Copyright (c) 2014 IEEE. Reprinted from "Wu, Y., Zhu, Q., Huang, J. \& Tsang, D.H.K. (2014). Revenue sharing based resource allocation for dynamic spectrum access networks. IEEE Journal on Selected Areas in Communications, v. 32 (11), Article number 6985751, p. 2280-2296."

This material is posted here with permission of the IEEE. Such permission of the IEEE does not in any way imply IEEE endorsement of any of The Hong Kong University of Science and Technology's products or services. Internal or personal use of this material is permitted. However, permission to reprint/republish this material for advertising or promotional purposes or for creating new collective works for resale or redistribution must be obtained from the IEEE by writing to pubspermissions@ieee.org.

By choosing to view this document, you agree to all provisions of the copyright laws protecting it. 


\title{
Revenue Sharing based Resource Allocation for Dynamic Spectrum Access Networks
}

\author{
Yuan Wu, Qionghua Zhu, Jianwei Huang, Danny H.K. Tsang
}

\begin{abstract}
We propose a revenue sharing based resource allocation scheme for dynamic spectrum access (DSA) networks. In our scheme, based on a mutually agreed revenue sharing scheme, a primary network operator (PNO) actively shares its radio resource with a secondary network operator (SNO), which provides access service to secondary users (SUs) for its revenue maximization. To investigate the coupling effect between the revenue sharing and resource allocation, we formulate the interaction between PNO and SNO as a two-layered game, which includes a top layer game to model their revenue sharing and a bottom layer game to model their joint resource allocations. Specifically, in the top layer, based on their joint resource allocation decisions, the PNO and SNO form a Nash bargaining game to determine the revenue sharing scheme such that both of them can benefit from cooperation satisfactorily. Then, in the bottom layer, under the given revenue sharing scheme, the PNO and SNO form a Stackelberg game to determine their joint resource allocation decisions, which also influence their respective revenues. The two games work iteratively such that the PNO and SNO reach a final equilibrium state at which neither PNO nor SNO will change its decisions unilaterally in both layers. We propose efficient algorithms to solve both the top layer and bottom layer games and compute the final equilibrium of the two-layered game. Specifically, despite the non-convexity of joint resource allocation optimization problem in the bottom layer, we identify its hidden monotonic structure and propose an efficient algorithm, which is based on the polyblock approximation, to achieve the optimal solutions. Moreover, in the top layer, to tackle with the difficulty due to the lack of an analytical objective function for the revenue sharing problem, we explore its hidden unimodal property and propose a Brent's method based algorithm to achieve the optimal solution. Numerical results are presented to verify the performance of our algorithms and show that our revenue sharing based resource allocation scheme yields a winwin situation for the PNO and SNO.
\end{abstract}

\section{INTRODUCTION}

Dynamic spectrum access (DSA) is a new paradigm that can effectively improve spectrum utilization efficiency and alleviate spectrum congestion for the increasingly crowded wireless communication systems [1]-[5]. In DSA, the secondary unlicensed users (usually called the SUs) are allowed to opportunistically exploit the spectrum resources currently underutilized by the primary licensed users (usually called the

Manuscript received date: Jan 4, 2014; Revised date: May 6, 2014. This work was presented in part at the IoT Workshop of IEEE ICCC'2013 [30].

Y. Wu and Q. Zhu are with College of Information Engineering, Zhejiang University of Technology, Hangzhou, P.R. China (email: iewuy@zjut.edu.cn, qionghua_zhu@163.com). Y. Wu is also with the State Key Laboratory of Integrated Services Networks, Xidian University, Xian 710162, China.

J. Huang is with the Network Communications and Economics Lab, Department of Information Engineering, The Chinese University of Hong Kong, Hong Kong (e-mail: jwhuang@ie.cuhk.edu.hk).

D. H.K. Tsang is with Department of Electronic and Computer Engineering, Hong Kong University of Science and Technology, Hong Kong (email: eetsang@ee.ust.hk).
PUs). The success of DSA requires the SUs to intelligently exploit the underutilized spectrum and yield an unharmful (or controllable) interference to the PUs. The prior literature on resource allocation of DSA can be categorized by the roles of PUs, namely, the passive PU model and the active PU model. The passive model assumes that a PU is unaware of the operations of SUs (who are usually obligated to perform spectrum sensing to explore idle spectrum), and it does not require any modification for the PU systems [6]-[8]. Despite its advantage of being backward compatible with the legacy communication systems nowadays, the passive model gains the PU nothing. In contrast, in the active model, a PU is assumed to know the existence of SUs and can benefit from DSA by leasing its spectrum resources to the SUs, e.g., obtaining either an improved transmission performance [9]-[11] or economic compensations [12]-[17], [19]-[26]. Specifically, in this paper, we consider the active PU model, in which the primary network operator aims at obtaining an economic gain by leasing its radio resource to the secondary network operator.

There are several different economic mechanisms that can coordinate the interactions between the PUs and SUs in the active PU model. Next we review two commonly used approaches: pricing and auction. Pricing strategy is an effective strategy to motivate the resource leasing of primary network [15]-[18]. Specifically, the authors of [15] analyzed competitive and cooperative pricing of the PUs to lease their temporarily idle bandwidth to the SUs. The authors of [16] further investigated a multi-level marketed model, where the PUs sold the idle bandwidth to the SUs, which again sold the allocated bandwidth to tertiary and quaternary services. Recent paper [17] proposed a model in which the PUs charged the SUs for their time-slot leasing. Besides leasing the idle resource to the SUs in an interference-free manner, the PUs can also allow concurrent transmission of the SUs and then charge the suffered interference from them. Related papers [19]-[22] investigated the charging of SUs' interference to optimize the revenue of PU. Specifically, in [19], [20], the PU aimed at maximizing its revenue by charging the SUs' interference, which was subject to a fixed interference-cap. In comparison, in [21], [22], the PU's interference-cap was considered to be a tunable parameter, which worked together with the power allocation to optimize the revenue of PU. Auction strategy is also an effective strategy to motivate the resource leasing of primary network [23]-[27]. Specifically, the authors of [23] proposed a multi-auctioneer progressive auction to model the spectrum sharing of multiple PUs. In [24], the authors investigated a short term secondary spectrum trading between one PU and multiple SUs in a hybrid spectrum market to optimize the expected profit of PU. Recent paper [25] adopted 
the auction strategy to jointly maximize the total satisfaction of all SUs as well as the revenue of PU. Different from [23]-[25] which considered the interference-free model and treated the idle spectrum as a tradable commodity, the authors of [26], [27] considered the interference-cap as a commodity for trading in auction.

Different from the approaches of pricing and auction, we propose a revenue sharing based resource allocation scheme for the primary network operator (PNO) and secondary network operator (SNO). In our scheme, a PNO, which provides access service to the PU, leases part of its spectrum resources to a SNO, which again uses the leased resources to provide access service to the SUs. The SNO then shares part of the corresponding revenue with the PNO. The revenues achieved by the PNO and SNO depend on both their revenue sharing scheme and their joint resource allocation decisions, which are coupled together. Intuitively, a revenue sharing scheme which favors the SNO will discourage the PNO to lease its spectrum resources to the SNO, which in turn reduces the SNO's revenue. However, a revenue sharing scheme which favors the PNO will directly reduce the revenue of SNO. Hence, it is important to achieve a proper balance between the revenue sharing and the joint resource allocations to achieve a win-win situation for both PNO and SNO.

There are several recent papers that investigated the bandwidth sharing among network service providers by using a revenue (or cost) sharing economic mechanism [28], [29]. Differing from the orthogonal bandwidth sharing in [28], [29], the joint resource allocation decisions of PNO and SNO in our model take account of the co-channel interferences between them, which thus lead to non-convex resource allocation problems in the bottom layer. Furthermore, due to the fact that the optimal joint resource allocation decisions cannot be derived analytically, the bargaining based revenue sharing between PNO and SNO in the top layer again yields a complicated optimization problem whose objective function cannot be easily characterized. Solving these coupled and nonconvex optimization problems efficiently is a key contribution of this paper. Our main contributions in this paper can be summarized as follows.

- To investigate the proper tradeoff between revenue sharing and resource allocation, we model the interaction between PNO and SNO as a two-layered game. First, in the top layer, based on the joint resource allocation decisions, the PNO and SNO form a Nash bargaining game to reach an agreement on their revenue sharing such that both of them can benefit [44]. Second, in the bottom layer, under a given revenue sharing scheme, the PNO and SNO form a Stackelberg game, a two-stage dynamic game, to reach an equilibrium in their joint resource allocation decisions [45]. The two games work iteratively to reach the final equilibrium, at which neither the PNO nor the SNO will change its decisions unilaterally in both layers.

- We propose algorithms to compute the equilibrium of the two-layered game. We first characterize the optimality condition for the SNO's revenue maximization problem. Using this condition, we reveal the monotonic structure of the PNO's revenue maximization problem and propose efficient algorithms to determine the optimal solutions, which subsequently lead to the optimal joint resource allocation decisions for the PNO and SNO in the bottom layer. Based on this, we further propose an efficient algorithm to derive the optimal revenue sharing scheme between the PNO and SNO in the top layer.

- Extensive numerical results demonstrate the accuracy and computational efficiency of our proposed algorithms. We also show that our resource allocation scheme leads to a win-win situation for the PNO and SNO and enables them to positively benefit in a fair manner.

The rest of this paper is organized as follows. We discuss the system model and problem formulation in Section II. We derive the optimal joint resource allocation decisions in the bottom layer in Section III and Section IV. We then derive the optimal revenue sharing scheme in the top layer in Section V. We present the numerical results in Section VI and conclude this work in Section VII.

\section{System Model And Problem Formulation}

We first describe the system model in Subsection II-A. Then, we present the mathematical problem formulations in Subsection II-B and Subsection II-C.

\section{A. System Model}

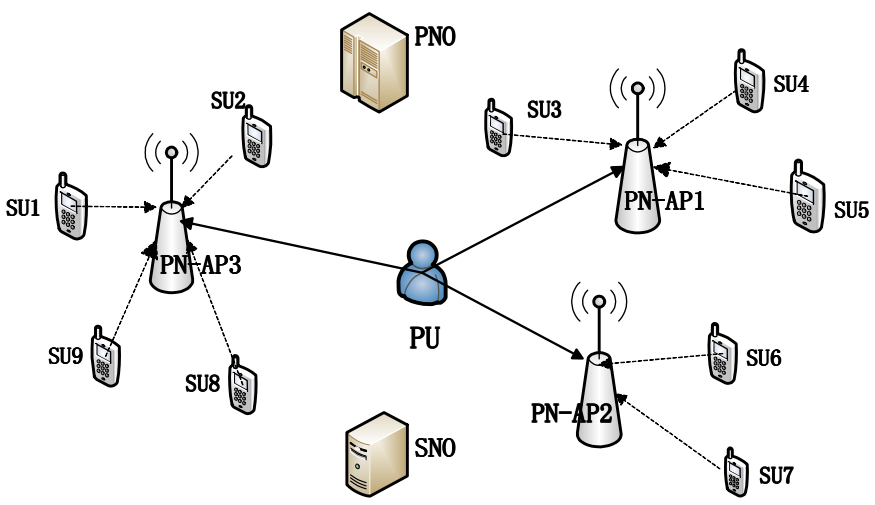

Fig. 1: An illustrative system model. A PNO uses three APs to provide uplink access service to the PU. The PNO leases its APs to a SNO, which provides access service to the SUs (by using the leased APs from the PNO). Around each AP $k$, there is a group of SUs $\mathcal{S}_{k}$, who, under the control of SNO, access AP $k$ for their uplink transmission.

As illustrated in Figure 1, in our system model, the PNO uses a set $\mathcal{K}=\{1,2, \ldots, K\}$ of APs to provide uplink access service to a representative PU. Each AP $k$ is allocated a licensed bandwidth of $B^{k}$, and different APs operate in non-overlapping bandwidths. In the rest of the paper, we treat AP $k$ and its equipped spectrum (or channel) $k$ interchangeably. The PNO can adjust the uplink transmission rates of PU on its different APs (i.e., from the PU to the APs) with a guarantee of the PU's quality of service (QoS) requirements. Meanwhile, there also exists a SNO that coexists with the PNO and provides uplink access service to the SUs by renting the PNO's APs. Specifically, around each AP $k$, there exists a group of SUs, who, under the control of SNO, access AP $k$ for their uplink transmission (i.e., from the SUs to AP $k$ ). We denote this 
set of SUs by $\mathcal{S}_{k}=\left\{S_{1}, S_{2}, \ldots, S_{M_{k}}\right\}$ with $M_{k}$ denoting the total number of SUs belonging to set $\mathcal{S}_{k}{ }^{1}$. To lease its APs to the SNO, the PNO controls the interference-cap $Q_{0}^{k}$ at each AP $k$, which represents the PNO's tolerable interference level measured at AP $k$. Subject to the PNO's interference-caps, the SNO then determines each SU's bandwidth allocation and transmit-power to maximize its revenue from providing the access service. Meanwhile, as a compensation for its lease of APs, the PNO is allowed to share part of the SNO's revenue. Let $\omega$ denote the sharing factor, which represents the portion of SNO's total revenue shared to the PNO. Specifically, $\omega=1$ means that the PNO gets all the SNO's revenue and leaves the SNO nothing. In contrast, $\omega=0$ means that the PNO gets nothing from the SNO, implying that the PNO and SNO fail to reach an agreement on their revenue sharing.

The revenues achieved by the PNO and SNO depend on both their agreed revenue sharing scheme and their joint resource allocation decisions. Therefore, to quantify their respective revenues, we need to take account of the following two-layered interaction (the details are shown in Figure 2):

- In the bottom layer, based on a given sharing factor $\omega$, the PNO and SNO determine their joint resource allocation decisions to maximize their respective revenues. Since the PNO is dominant in leasing its APs, the PNO (as the leader) first determines its interference-caps $\left\{Q_{0}^{k}\right\}$ at its APs and the PU's transmit-power $\left\{p_{0}^{k}\right\}$ (to meet the PU's QoS requirements). After knowing the PNO's decisions, the SNO (as the follower) determines each SU's bandwidth allocation and transmit-power. This leader-follower interaction is modeled as a Stackelberg game, whose equilibrium corresponds to the optimal joint resource allocation decisions of PNO and SNO in the bottom layer.

- In the top layer, based on their joint resource allocation decisions, the PNO and SNO determine the sharing factor such that both of them can achieve satisfactory revenues. This process is modeled as a Nash bargaining game, whose equilibrium specifies the optimal sharing factor for the PNO and SNO.

In the next two subsections, we will present the mathematical formulations for each layer of the game.

\section{B. Stackelberg Game in Bottom Layer for Joint Resource Allocations}

In this subsection, we mathematically formulate the Stackelberg game in the bottom layer, which includes the game leader PNO's optimization problem and the game follower SNO's optimization problem. We will analyze the Stackelberg game through a backward induction, starting from the SNO's optimization problem.

1) SNO's Optimization Problem: Knowing the PNO's decisions on its interference-caps and the PU's transmit-power, the SNO determines each SU's bandwidth allocation and transmitpower to maximize its revenue.

\footnotetext{
${ }^{1}$ In this work, we assume that each SU has one radio interface and thus can only access the nearest AP to it. Hence, we have $\mathcal{S}_{k} \cap \mathcal{S}_{j}=\emptyset$ when $k \neq j$ and $k, j \in \mathcal{K}$. Notice that related work [19] also adopted a similar model in which the SNO can use the APs of PNO for access service.
}

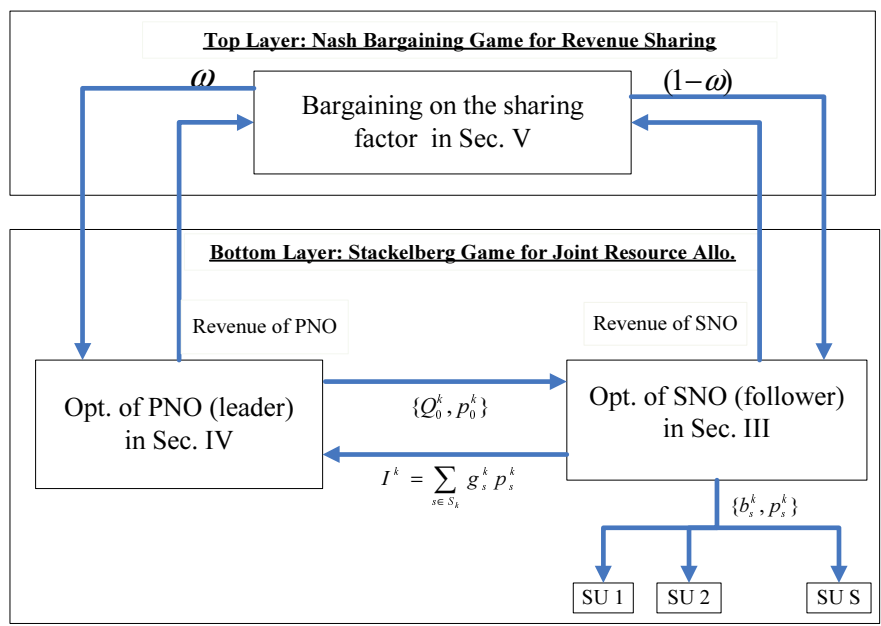

Fig. 2: A two-layered interaction. The bottom block: the PNO and SNO form a Stackelberg game to determine the joint resource allocation decisions. The top block: the PNO and SNO form a Nash bargaining game to determine the revenue sharing scheme.

Specifically, let $b_{s}^{k}$ and $p_{s}^{k}$ denote SU s's allocated bandwidth and transmit-power by the SNO on AP $k$, respectively. Then, the uplink throughput of SU $s$ via AP $k$ is given by

$$
R_{s}^{k}=b_{s}^{k} \log _{2}\left(1+\frac{g_{s}^{k} p_{s}^{k}}{b_{s}^{k}\left(n_{0}+g_{0}^{k} p_{0}^{k} / B^{k}\right)}\right) .
$$

Here, $n_{0}$ denotes the power density of the background noise. $g_{s}^{k}$ denotes the channel gain from SU $s$ to AP $k$, and $g_{0}^{k}$ denotes the channel gain from the PU to AP $k$. Recall that $p_{0}^{k}$ denotes the PU's transmit-power on AP $k$. Since the PU's transmission (i.e., from the PU to each AP) occupies the entire bandwidth of each AP $k$, the interference power density from the PU is thus given by $\frac{p_{0}^{k} g_{0}^{k}}{B^{k}}, \forall s \in \mathcal{S}_{k}$.

Using the access service controlled by SNO, the SUs make the corresponding payments to the SNO. Let $\rho$ denote the SNO's price (e.g., with the unit of $\$ / \mathrm{Mbps}$ ) charged to the throughput of each SU. Then, given the sharing factor $\omega$, the SNO's revenue maximization problem is formulated as follows

$$
\begin{aligned}
& \text { (SNO-P): } S(\omega)= \\
& \max (1-\omega) \rho \sum_{k \in \mathcal{K}} \sum_{s \in \mathcal{S}_{k}} b_{s}^{k} \log _{2}\left(1+\frac{g_{s}^{k} p_{s}^{k}}{b_{s}^{k}\left(n_{0}+g_{0}^{k} p_{0}^{k} / B^{k}\right)}\right) \\
& \text { subject to: } \sum_{s \in \mathcal{S}_{k}} b_{s}^{k} \leq B^{k}, \forall k \in \mathcal{K}, \\
& I^{k}=\sum_{s \in \mathcal{S}_{k}} g_{s}^{k} p_{s}^{k} \leq Q_{0}^{k}, \forall k \in \mathcal{K}, \\
& p_{s}^{k} \leq P_{s}^{\max }, \forall s \in \mathcal{S}_{k}, \forall k \in \mathcal{K}, \\
& \text { variables: }\left\{b_{s}^{k}, p_{s}^{k}\right\}_{\forall s \in \mathcal{S}_{k}, \forall k \in \mathcal{K} .}
\end{aligned}
$$

Constraint (2) means that for each $\mathrm{AP} k$, the total bandwidth allocated by the SNO should be no more than the total bandwidth $B^{k}$ of AP $k$. Constraint (3) means that for each AP $k$, the total interference caused by the SUs, i.e., $I^{k}=\sum_{s \in \mathcal{S}_{k}} g_{s}^{k} p_{s}^{k}$, should not violate the PNO's interference-cap $Q_{0}^{k}$. Although different SUs in $\mathcal{S}_{k}$ use different portions of AP $k$ 's bandwidth, they all cause interference to the PU, because the PU's transmission occupies the entire bandwidth of AP $k$. In particular, we 
assume that the PU treats the aggregate interference at AP $k$, which is from all SUs in $\mathcal{S}_{k}$, as a whole. Notice that such interference-cap constraints also appeared in [41], [42]. Finally, constraint (4) means that each SU s's transmit-power is no greater than its upper bound $P_{s}^{\max }$.

Our focuses in this work are to understand the collaboration between PNO and SNO and to investigate their potential benefits from it. To this end, in Problem (SNO-P), we assume that each SU always has sufficient data to send, and thus it will fully utilize the bandwidth allocation and transmit-power determined by the SNO and pay the SNO accordingly. This optimistic assumption corresponds to an upper bound of the network revenue.

2) PNO's Optimization Problem: Knowing the SNO's optimal bandwidth allocation and power allocation (i.e., the optimal solutions of Problem (SNO-P)), the PNO will determine its interference-caps $\left\{Q_{0}^{k}\right\}$ and the PU's transmit-power $\left\{p_{0}^{k}\right\}$ to maximize its revenue.

First, the PU's transmit-power should meet its own QoS requirements represented below

$$
\begin{array}{r}
R_{0}^{k, \min } \leq B^{k} \log _{2}\left(1+\frac{g_{0}^{k} p_{0}^{k}}{n_{0} B^{k}+I^{k}}\right) \leq R_{0}^{k, \max }, \forall k \in \mathcal{K}, \\
\sum_{k \in \mathcal{K}} B^{k} \log _{2}\left(1+\frac{g_{0}^{k} p_{0}^{k}}{n_{0} B^{k}+I^{k}}\right) \geq R_{0}^{\text {req }} .
\end{array}
$$

Here, $R_{0}^{k, \min }$ and $R_{0}^{k, \max }$ denote the minimum rate and the maximum rate of $\mathrm{PU}$ at $\mathrm{AP} k$, respectively, to guarantee a successful transmission. Besides, $R_{0}^{\text {req }}$ denotes the PU's total throughput requirement, which satisfies $\sum_{k} R_{0}^{k, \min } \leq$ $R_{0}^{\text {req }} \leq \sum_{k} R_{0}^{k, \max }$. Recall that the PNO treats the aggregate interference from all SUs in $\mathcal{S}_{k}$ as a whole, thus yielding the PU's uplink throughput on each AP $k$ as shown in the above constraints (5) and (6). Due to this co-channel interference, the PU needs to consume additional transmitpower to guarantee its throughput requirements, compared to the case without allowing any interference from the SUs (i.e., $\left.Q_{0}^{k}=0, \forall k\right)$. Let $\alpha$ denote the PU's marginal cost for its power consumption. Thus, the PU's additional power cost is given by $\alpha\left(\sum_{k \in \mathcal{K}} p_{0}^{k}-\bar{p}_{0}\right)$. Here, $\bar{p}_{0}$ denotes the PU's minimum transmit-power to guarantee its throughput requirements, and its value can be given by

$$
\bar{p}_{0}=\min _{\left\{p_{0}^{k}\right\}_{k \in \mathcal{K}}} \sum_{k \in \mathcal{K}} p_{0}^{k},
$$

subject to: $I^{k}=0, \forall k \in \mathcal{K}$, and constraints $(5),(6)$.

We treat $\bar{p}_{0}$ as a constant in the rest of the paper. In this work, we consider that the set of APs, which are owned by the PNO, are connected with the controller (or central server) of PNO via the wired networks (e.g., an optical fiber network). Thus, the APs can measure the channel state information and then send such information to the PNO for its subsequent radio resource allocations. This is similar to the current practice in base-station subsystems (BSS) of cellular networks, where several neighboring base-transceiver stations (BTSs) are connected with a common base station controller (BSC) via wired lines. The BTSs report the required information (e.g., channel information) to the BSC, and operate under the control of
BSC to provide radio access to mobile users. Similarly to the PNO, we assume that the SNO is connected to the sets of APs via a wired network to obtain the channel state information between the APs and SUs. Furthermore, we consider a FDD system in which the APs first measure the uplink channel state information from the PU (or SUs) via some conventional pilot-based channel estimation schemes, and then feedback the consequent instructions on radio resource control to the PU (or SUs) for transmission ${ }^{2}$.

Considering the share of SNO's revenue, the PNO's revenue maximization problem, which takes account of the PU's additional power consumption cost, is as follows

$$
\begin{aligned}
& \text { (PNO-P): } F_{0}(\omega)= \\
& \max \omega \rho \sum_{k \in \mathcal{K}} \sum_{s \in \mathcal{S}_{k}} b_{s}^{k} \log _{2}\left(1+\frac{g_{s}^{k} p_{s}^{k}}{b_{s}^{k}\left(n_{0}+g_{0}^{k} p_{0}^{k} / B^{k}\right)}\right)- \\
& \alpha\left(\sum_{k \in \mathcal{K}} p_{0}^{k}-\bar{p}_{0}\right), \\
& \text { subject to: } I^{k}=\sum_{s \in S_{k}} g_{s}^{k} p_{s}^{k} \leq Q_{0}^{k}, \forall k \in \mathcal{K} \\
& \text { variables: }\left\{Q_{0}^{k}, p_{0}^{k}\right\}_{\forall k \in \mathcal{K} .}
\end{aligned}
$$

The objective function of Problem (PNO-P) represents the PNO's goal of maximizing its additional profit from resource sharing, i.e., the difference between its shared revenue from the SNO and the PU's additional power consumption cost to meet its throughput requirements. In other words, the larger the shared revenue and the lower the cost the better. Hence, these two terms are parts of the objective function. Meanwhile, the PNO only needs to provide a required throughput for the PU to meet its QoS, which motives us to adopt constraints (5) and (6). Notice that in Problem (PNO-P), the PNO faces an intrinsic tradeoff in setting its interference-caps. Specifically, setting the interference-caps tightly will reduce the PU's additional power consumption cost, but this will also reduce the SNO's capability of collecting revenue, which in turn reduces the PNO's revenue.

The equilibrium of bottom layer game corresponds to the joint resource allocation decisions, including i) the PNO's decisions on its interference-caps and the PU's transmit-power, which are the optimal solutions of Problem (PNO-P), and ii) the SNO's decisions on each SU's bandwidth allocation and transmit-power, which are the optimal solutions of Problem (SNO-P).

\section{Nash Bargaining in Top Layer for Revenue Sharing Scheme}

In this subsection, we mathematically formulate the Nash bargaining game in the top layer.

\footnotetext{
${ }^{2}$ As a first attempt to model and understand the intrinsic coupling effect between the revenue sharing and cooperative radio resource allocation for the primary and secondary networks, in this work, we focus on the scenario of quasi-static channel environment, where the channel state keeps unchanged or changes slowly during the time frame of interests (e.g., transmission duration for one data frame). In practice, the channel information might be imperfect, e.g. being inaccurate and outdated. Incorporating the impact of imperfect channel information requires to formulate the optimization problem via the robust optimization techniques as in [38]-[40], and it is an important future direction to extend this work.
} 
Based on the optimal joint resource allocation decisions in the bottom layer, the PNO knows its revenue $F_{0}(\omega)$, which is the optimal objective value of Problem (PNO-P), and the SNO knows its revenue $S(\omega)$, which is the optimal objective value of Problem (SNO-P). Based on $F_{0}(\omega)$ and $S(\omega)$, the PNO and SNO further negotiate about the sharing factor $\omega$ to achieve a satisfactory win-win situation, as shown in the top block in Figure 2. This negotiation process essentially matches the practice that two agents of different interests bargain over a social yet limited resource, which thus motivates us to model it as a Nash bargaining problem as follows (the capital letters "RS" stand for "Revenue Sharing"):

(RS-P): $\max _{0 \leq \omega \leq 1} W(\omega)=\left(F_{0}(\omega)-F_{0}(0)\right)(S(\omega)-S(0))$.

Here, $F_{0}(0)$ and $S(0)$ denote the respective benchmark revenues of PNO and SNO, when they fail to reach an agreement. Problem (RS-P) aims to ensure that both PNO and SNO can positively benefit from cooperation beyond their benchmark performance in a fair manner.

In the next Section III and Section IV, we solve Problem (SNO-P) and Problem (PNO-P), respectively, thus reaching the equilibrium in the bottom layer. Then, in Section $\mathrm{V}$, we solve Problem (RS-P) in the top layer.

\section{Solving the Bottom Layer Game: THE SNO'S OPTIMIZATION}

We use the backward induction to solve the Stackelberg game in the bottom layer. In this section, we first solve the SNO's Problem (SNO-P), which is repeated below, under the given PNO's decisions $\left\{Q_{0}^{k}, p_{0}^{k}\right\}$ and the sharing factor $\omega$ :

(SNO-P):

$\max (1-\omega) \rho \sum_{k \in \mathcal{K}} \sum_{s \in \mathcal{S}_{k}} b_{s}^{k} \log _{2}\left(1+\frac{g_{s}^{k} p_{s}^{k}}{b_{s}^{k}\left(n_{0}+g_{0}^{k} p_{0}^{k} / B^{k}\right)}\right)$

subject to: constraints (2), (3) and (4),

variables: $\left\{b_{s}^{k}, p_{s}^{k}\right\}_{\forall s \in \mathcal{S}_{k}, \forall k \in \mathcal{K} \text {. }}$

We can characterize Problem (SNO-P) as follows.

Lemma 1: (Convexity of Problem (SNO-P)) Problem (SNO-P) is a jointly convex optimization problem with respect to $\left\{b_{s}^{k}\right\}$ and $\left\{p_{s}^{k}\right\}$.

Proof: It is easy to show that the Hessian matrix of each component, i.e., $\log _{2}\left(1+\frac{g_{s}^{k} p_{s}^{k}}{b_{s}^{k}\left(n_{0}+g_{0}^{k} p_{0}^{k} / B^{k}\right)}\right)$, is negatively definite with respect to $\left\{b_{s}^{k}, p_{s}^{k}\right\}$ (the detailed calculation can be referred to [31] and is omitted here for space limitation). Further because constraints (2)-(4) are all linear functions, Problem (SNO-P) is a convex problem [43].

Let $\left\{b_{s}^{k *}\right\}$ and $\left\{p_{s}^{k *}\right\}$ denote the SNO's optimal decisions on the bandwidth allocations and transmit-power for Problem (SNO-P), respectively. We have the following result.

Lemma 2: (Structural Property of SNO's Optimal Resource Allocation Decisions) The optimal solutions of Problem (SNOP) meet the following condition for each AP $k$,

$$
b_{s}^{k *}=B^{k} \frac{g_{s}^{k} p_{s}^{k *}}{I^{k *}}, \forall s \in \mathcal{S}_{k},
$$

where $I^{k *}=\sum_{s \in \mathcal{S}_{k}} g_{s}^{k} p_{s}^{k *}$ denotes the aggregate interference to AP $k$ under the SNO's optimal power allocations.
Proof: Please refer to Appendix I. Notice that the above result holds even when $p_{s}^{k *}=0$ for some SU $s$.

Lemma 2 means that the optimal bandwidth allocation to each SU should be proportional to its interference incurred. We further replace $b_{s}^{k}$ by using (7), and thus express SU $s$ 's throughput at AP $k$ as follows

$$
\begin{aligned}
R_{s}^{k} & =\frac{g_{s}^{k} p_{s}^{k}}{I^{k}} B^{k} \log _{2}\left(1+\frac{I^{k}}{n_{0} B^{k}+g_{0}^{k} p_{0}^{k}}\right) \\
& =\frac{g_{s}^{k} p_{s}^{k}}{\sum_{s \in \mathcal{S}_{k}} p_{s}^{k} g_{s}^{k}} B^{k} \log _{2}\left(1+\frac{\sum_{s \in \mathcal{S}_{k}} p_{s}^{k} g_{s}^{k}}{n_{0} B^{k}+g_{0}^{k} p_{0}^{k}}\right) .
\end{aligned}
$$

Then, the original Problem (SNO-P) can be equivalently transformed into the following problem, which only depends on the SUs' transmit-power (the capital letter "E" stands for "Equivalence")

(SNO-P-E): $\max (1-\omega) \rho \sum_{k \in \mathcal{K}} B^{k} \log _{2}\left(1+\frac{\sum_{s \in \mathcal{S}_{k}} p_{s}^{k} g_{s}^{k}}{n_{0} B^{k}+g_{0}^{k} p_{0}^{k}}\right)$

subject to: $\sum_{s \in \mathcal{S}_{k}} p_{s}^{k} g_{s}^{k} \leq Q_{0}^{k}, \forall k$,

$$
p_{s}^{k} \leq P_{s}^{\max }, \forall s \in \mathcal{S}_{k} .
$$

variables: $\left\{p_{s}^{k}\right\}_{\forall s \in \mathcal{S}_{k}, \forall k \in \mathcal{K}}$

Remark 1: The objective function of Problem (SNO-P-E) indicates that the SNO's revenue increases in the total interference at each AP (i.e., $I^{k}=\sum_{s \in \mathcal{S}_{k}} p_{s}^{k} g_{s}^{k}$ ), which is consistent with the intuitions. Suppose that $\sum_{s \in \mathcal{S}_{k}} P_{s}^{\max } g_{s}^{k} \geq Q_{0}^{k}$ (which is a reasonable assumption from a practical point of view, since the PNO will gain no more revenue by setting its interference-cap $\left.Q_{0}^{k}>\sum_{s \in \mathcal{S}_{k}} P_{s}^{\max } g_{s}^{k}\right)$. Then, we always have $I^{k}=Q_{0}^{k}, \forall k$ at the optimal solutions of Problem (SNO-P-E). In other words, to maximize its revenue, the SNO should fully utilize the interference-caps at all APs.

Although we know the optimality condition of Problem (SNO-P) in Lemma 2, the optimal bandwidth allocation and transmit-power for each SU still need to be determined. For this purpose, we propose the following fairness rule, which does not affect the SNO's maximum revenue but leads to a fair resource allocation among all SUs accessing the same AP. Specifically, let $\beta_{s}$ denote the weight associated with SU $s$ (notice that our analysis below applies to arbitrary choice of positive weights $\left\{\beta_{s}\right\}$ of SUs). Then, the fairness rule requires the following condition to hold

$$
\frac{R_{s}^{k}}{\beta_{s}}=\frac{R_{j}^{k}}{\beta_{j}}, \forall s \neq j \text { and } s, j \in \mathcal{S}_{k} .
$$

Based on eq. (12) above, we have the following results.

Lemma 3: (SNO's Optimal Decisions on Bandwidth Allocation and Transmit-Power) Using the fairness rule in (12), the SNO's optimal decisions on the transit-power and bandwidth allocation for Problem (SNO-P-E) (and also for Problem (SNO-P) equivalently) are respectively given by

$$
\begin{aligned}
p_{s}^{k *} & =\frac{\beta_{s}}{\sum_{j} \beta_{j}} \frac{Q_{0}^{k}}{g_{s}^{k}}, \forall s \in \mathcal{S}_{k}, \forall k \in \mathcal{K}, \\
b_{s}^{k *} & =\frac{\beta_{s}}{\sum_{j} \beta_{j}} B^{k}, \forall s \in \mathcal{S}_{k}, \forall k \in \mathcal{K} .
\end{aligned}
$$


Proof: Please refer to Appendix II. Notice that the resource allocations given by eq. (13) and eq. (14) guarantee that the SNO can fully exploit the PNO's interference-caps at all APs. Therefore, they will not yield any loss to the SNO's maximum revenue according to our previous discussion in Remark 1.

\section{Solving the Bottom Layer Game: THE PNO'S OPTIMIZATION}

After knowing the SNO's optimal bandwidth allocation and power allocation to each $\mathrm{SU}$, in this section, we continue to solve Problem (PNO-P). In particular, we will solve Problem (PNO-P) via the following three procedures.

- Procedure-I: We first make a series of equivalent transformations of Problem (PNO-P), and then identify the hidden convexity of the subsequently obtained optimization problem. Based on the hidden convexity, we obtain a structural property of the PNO's optimal power allocation as a function of its rate allocation.

- Procedure-II: Using the structural property from Procedure-I, we equivalently transform Problem (PNO-P) into a PNO's rate allocation problem. We further identify the monotonic structure of this rate allocation problem.

- Procedure-III: Using the monotonic structure from Procedure-II, we propose efficient algorithms to obtain the PNO's optimal rate allocation and the corresponding optimal resource allocations.

Next, we illustrate the details of these three procedures.

A. Procedure-I: Equivalent Transformations of PNO's Optimization Problem and its Hidden Property

1) Equivalent Transformations of PNO's Optimization: Using the property that the SNO always makes $I^{k}=Q_{0}^{k}, \forall k$ (as described in Remark 1) and putting $\sum_{s \in \mathcal{S}_{k}} R_{s}^{k}$ in eq. (8) into Problem (PNO-P), we obtain an equivalent form of Problem (PNO-P) as follows (the capital letter "E" stands for "Equivalence")

(PNO-P-E):

$\max \omega \rho \sum_{k \in \mathcal{K}} B^{k} \log _{2}\left(1+\frac{Q_{0}^{k}}{n_{0} B^{k}+g_{0}^{k} p_{0}^{k}}\right)-\alpha\left(\sum_{k \in \mathcal{K}} p_{0}^{k}-\overline{p_{0}}\right)$

subject to:

$R_{0}^{k, \text { min }} \leq B^{k} \log _{2}\left(1+\frac{g_{0}^{k} p_{0}^{k}}{n_{0} B^{k}+Q_{0}^{k}}\right) \leq R_{0}^{k, \max }, \forall k \in \mathcal{K}(, 15)$

$\sum_{k \in \mathcal{K}} B^{k} \log _{2}\left(1+\frac{g_{0}^{k} p_{0}^{k}}{n_{0} B^{k}+Q_{0}^{k}}\right) \geq R_{0}^{\text {req }}$,

variables: $\left\{p_{0}^{k}, Q_{0}^{k}\right\}_{\forall k \in \mathcal{K}}$.

Notice that Problem (PNO-P-E) is always feasible since the PNO can set its interference-cap to be zero, i.e., completely denying the SUs' access. Thus, the PU's QoS requirements can be satisfied for sure. Recall that we impose $\sum_{k} R_{0}^{k, \min } \leq$ $R_{0}^{\text {req }} \leq \sum_{k} R_{0}^{k, \text { max }}$, as stated in the paragraph after eq. (6).

Problem (PNO-P-E) is still a complicated and non-convex optimization problem, since the objective function and its constraint (16) are not jointly convex with respect to $\left\{p_{0}^{k}, Q_{0}^{k}\right\}$. To solve it, we introduce the auxiliary variable $R_{0}^{k}=$
$B^{k} \log _{2}\left(1+\frac{g_{0}^{k} p_{0}^{k}}{n_{0} B^{k}+Q_{0}^{k}}\right), \forall k \in \mathcal{K}$, which represents the PNO's rate allocation to the PU at AP $k$. Thus, the interference-cap $Q_{0}^{k}$ can be expressed in the terms of the rate allocation and transmit-power as follows

$$
Q_{0}^{k}\left(R_{0}^{k}, p_{0}^{k}\right)=\frac{g_{0}^{k} p_{0}^{k}}{2^{R_{0}^{k} / B^{k}}-1}-n_{0} B^{k}, \forall k \in \mathcal{K} .
$$

By substituting the above result into Problem (PNO-P-E) and further performing some manipulations, we obtain an equivalent form of Problem (PNO-P-E) as follows

$$
\begin{gathered}
\text { (PNO-P-E') } \max _{\left\{p_{0}^{k}, R_{0}^{k}\right\}_{\forall k \in \mathcal{K}}} \omega \rho \sum_{k \in \mathcal{K}} B^{k} \log _{2} \frac{2^{R_{0}^{k} / B^{k}}}{\left(2^{R_{0}^{k} / B^{k}}-1\right)}+ \\
\omega \rho \sum_{k \in \mathcal{K}} B^{k} \log _{2}\left(\frac{g_{0}^{k} p_{0}^{k}}{n_{0} B^{k}+g_{0}^{k} p_{0}^{k}}\right)-\alpha\left(\sum_{k \in \mathcal{K}} p_{0}^{k}-\bar{p}_{0}\right)
\end{gathered}
$$

subject to: $g_{0}^{k} p_{0}^{k} \geq\left(2^{R_{0}^{k} / B^{k}}-1\right) n_{0} B^{k}, \forall k \in \mathcal{K}$,

$$
\begin{aligned}
& R_{0}^{k, \text { min }} \leq R_{0}^{k} \leq R_{0}^{k, \max }, \forall k \in \mathcal{K}, \\
& \sum_{k \in \mathcal{K}} R_{0}^{k} \geq R_{0}^{\text {req }} .
\end{aligned}
$$

The new constraint (18) guarantees that the original interference-cap is nonnegative at each AP.

2) Hidden Convexity and Structural Property of PNO's Power Allocation: Problem (PNO-P-E') is still non-convex in $\left\{R_{0}^{k}, p_{0}^{k}\right\}$, and thus is difficult to solve. A deeper investigation on it shows the following property.

Lemma 4: (Hidden Convexity of Problem (PNO-P-E')) Suppose that the rate allocation variables $\left\{R_{0}^{k}\right\}$, which are feasible to conditions (19) and (20), are fixed. Then, Problem (PNO-P-E') becomes an optimization problem with respect to the transmit-power $\left\{p_{0}^{k}\right\}$ only, and this problem is a strictly convex optimization problem separable for each AP.

Proof: Please refer to Appendix III.

Using Lemma 4, we first determine the optimal transmitpower as a function of the feasible rate allocation. Specifically, under a given $\left\{R_{0}^{k}\right\}$, the PNO's revenue maximization problem with respect to $\left\{p_{0}^{k}\right\}$ becomes

$$
\begin{aligned}
& \max _{\left\{p_{0}^{k}\right\}_{\forall k \in \mathcal{K}}} \omega \rho \sum_{k \in \mathcal{K}} B^{k} \log _{2} \frac{g_{0}^{k} p_{0}^{k}}{n_{0} B^{k}+g_{0}^{k} p_{0}^{k}}-\alpha\left(\sum_{k \in \mathcal{K}} p_{0}^{k}-\bar{p}_{0}\right) \\
& \text { subject to: } g_{0}^{k} p_{0}^{k} \geq\left(2^{R_{0}^{k} / B^{k}}-1\right) n_{0} B^{k}, \forall k \in \mathcal{K} .
\end{aligned}
$$

According to Lemma 4, the above problem has a unique optimal solution, which can be characterized as follows.

Proposition 1: (Structural Property of Optimal TransmitPower) For each AP $k$, there exists a critical threshold, which is denoted by $\eta^{k}$ and given by

$$
\eta^{k}=B^{k}\left(\log _{2}\left(1+\sqrt{1+\frac{4 \omega \rho g_{0}^{k}}{\alpha n_{0} \ln 2}}\right)-1\right) .
$$

Knowing $\eta^{k}$ for each AP $k$, the PNO's optimal decision on the PU's transmit-power, as a function of its rate allocation $R_{0}^{k}$, is given by

$p_{0}^{k *}\left(R_{0}^{k}\right)= \begin{cases}\frac{n_{0} B^{k}}{2 g_{0}^{k}}\left(\sqrt{1+\frac{4 \omega \rho g_{0}^{k}}{\alpha n_{0} \ln 2}}-1\right), & \text { if } R_{0}^{k} \leq \eta^{k} \\ \frac{\left(2_{0}^{k} / B^{k}-1\right) n_{0} B^{k}}{g_{0}^{k}}, & \text { if } R_{0}^{k}>\eta^{k}\end{cases}$ 
Further based on eq. (17), the PNO's optimal interference-cap at each AP $k$ is

$$
Q_{0}^{k *}\left(R_{0}^{k}\right)= \begin{cases}\frac{g_{0}^{k} p_{0}^{k *}\left(R_{0}^{k}\right)}{2^{R_{0}^{k} / B^{k}}-1}-n_{0} B^{k}, & \text { if } R_{0}^{k} \leq \eta^{k}, \\ 0, & \text { if } R_{0}^{k}>\eta^{k} .\end{cases}
$$

Proof: Please refer to Appendix IV.

Remark 2: The optimal transmit-power in (23) can be interpreted as follows. For each AP $k$, there is a critical threshold $\eta^{k}$ associated with the PNO's rate allocation. Based on $\eta^{k}$, we have the following two cases.

- if $R_{0}^{k} \leq \eta^{k}$, then the PU's optimal transmit-power $p_{s}^{k *}$ is fixed at $p_{s}^{k *}=\frac{n_{0} B^{k}}{2 g_{0}^{k}}\left(\sqrt{1+\frac{4 \omega \rho g_{0}^{k}}{\alpha n_{0} \ln 2}}-1\right)$, which is independent of $R_{0}^{k}$. In this case, the PNO allows to tolerate the SUs' interference, i.e., it sets its interference-cap at AP $k$ as $Q_{0}^{k *}\left(R_{0}^{k}\right)=\frac{\frac{n_{0} B^{k}}{2}\left(\sqrt{1+\frac{4 \omega \rho g_{0}^{k}}{\alpha n_{0} \ln 2}}-1\right)}{2^{R_{0}^{k} / B^{k}}-1}-n_{0} B^{k}$, which is positive.

- if $R_{0}^{k}>\eta^{k}$, then the PU's optimal transmit-power $p_{s}^{k *}=$ $\frac{\left(2^{R_{0}^{k} / B^{k}}-1\right) n_{0} B^{k}}{g_{0}^{k}}$, which grows exponentially in $R_{0}^{k}$. In this case, the PNO does not allow to tolerate any interference from the SUs, i.e., it sets its interference-cap at $\mathrm{AP} k$ as $Q_{0}^{k *}\left(R_{0}^{k}\right)=0$.

To verify Proposition 1, the top and bottom plots in Figure 3 show the transmit-power and interference-cap (in the yaxis) as functions of the PU's rate allocation (in the x-axis), respectively. We plot the results under three different values of $\frac{\omega \rho}{\alpha}$, which are marked by the circles, stars and triangles, respectively. The results show that when the rate allocation is below $\eta^{k}$, the corresponding transmit-power is constant, and the interference-cap is positive. In contrast, as the rate allocation is above $\eta^{k}$, the interference-cap decreases to zero, and the corresponding transmit-power increases drastically. Similar results also appear in Figure 4, in which we consider three different channel gains.

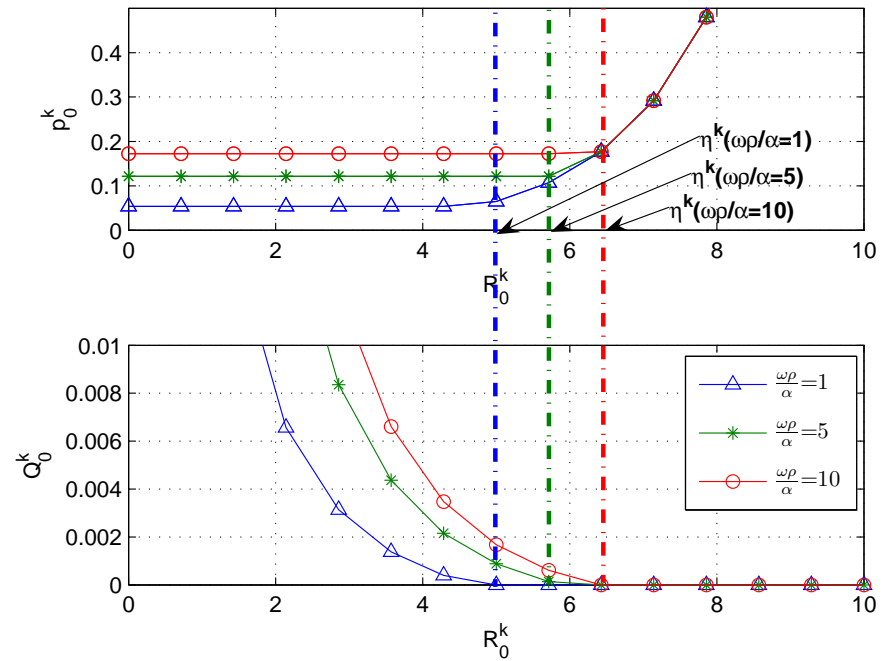

Fig. 3: The transmit-power and interference-cap as functions of $R_{0}^{k}$ under different values of $\frac{\omega \rho}{\alpha}$. Top plot: the optimal transmit-power. Bottom plot: the optimal interference-cap. We set $B^{k}=1 \mathrm{MHz}, g_{0}^{k}=$ 0.4813 , and $B^{k} n_{0}=0.001 \mathrm{~W}$.

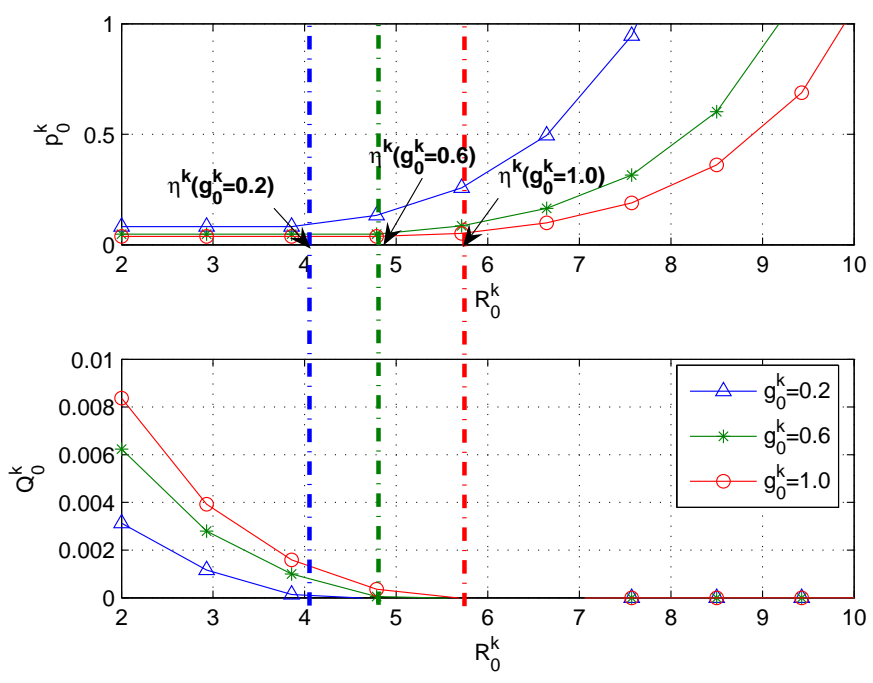

Fig. 4: The transmit-power and interference-cap as functions of $R_{0}^{k}$ under different $g_{0}^{k}$. Top plot: the PU's optimal transmit-power. Bottom plot: the PNO's optimal interference-cap. We set $\frac{\omega \rho}{\alpha}=1$.

\section{B. Procedure-II: PNO's Equivalent Rate Allocation Problem and its Monotonic Structure}

Based on Proposition 1, we define two different sets to categorize different APs, namely, $\Omega_{1}=\left\{k \mid R_{0}^{k, \text { min }} \leq R_{0}^{k} \leq\right.$ $\eta^{k}$, and $\left.k \in \mathcal{K}\right\}$ and $\Omega_{2}=\left\{k \mid \eta^{k} \leq R_{0}^{k} \leq R_{0}^{k, \max }\right.$, and $k \in$ $\mathcal{K}\}$. Notice that, to solve the original Problem (PNO-P-E'), we need to find the optimal combination of $\left(\Omega_{1}, \Omega_{2}\right)$. In particular, we first introduce a variable $C$, which depends on $\Omega_{1}$, as follows

$$
\begin{array}{r}
C\left(\Omega_{1}\right)=\sum_{k \in \Omega_{1}} \omega \rho B^{k} \log _{2}\left(\frac{\sqrt{1+\frac{4 \omega \rho g_{0}^{k}}{\alpha n_{0} \ln 2}}-1}{\sqrt{1+\frac{4 \omega \rho g_{0}^{k}}{\alpha n_{0} \ln 2}}+1}\right)- \\
\sum_{k \in \Omega_{1}} \alpha \frac{n_{0} B^{k}}{2 g_{0}^{k}}\left(\sqrt{1+\frac{4 \omega \rho g_{0}^{k}}{\alpha n_{0} \ln 2}}-1\right)+\alpha \bar{p}_{0} .
\end{array}
$$

Then, by substituting eq. (23) into the objective function of Problem (PNO-P-E'), we obtain a rate allocation problem, which is equivalent to Problem (PNO-P-E'), as follows

\section{(PNO-P-Rate):}

$$
\begin{gathered}
\max _{\left\{R_{0}^{k}\right\}_{\forall k \in \mathcal{K}, \Omega_{1}, \Omega_{2}}} \omega \rho \sum_{k \in \Omega_{1}} B^{k} \log _{2}\left(1+\frac{1}{2^{R_{0}^{k} / B^{k}}-1}\right)- \\
\alpha \sum_{k \in \Omega_{2}} \frac{\left(2^{R_{0}^{k} / B^{k}}-1\right) n_{0} B^{k}}{g_{0}^{k}}+C\left(\Omega_{1}\right) \\
\text { subject to: } R_{0}^{k, \text { min }} \leq R_{0}^{k} \leq \eta^{k}, \forall k \in \Omega_{1}, \\
\eta^{k} \leq R_{0}^{k} \leq R_{0}^{k, \max }, \forall k \in \Omega_{2}, \\
\sum_{k \in \Omega_{1}} R_{0}^{k}+\sum_{k \in \Omega_{2}} R_{0}^{k} \geq R_{0}^{\text {req }} .
\end{gathered}
$$

In Problem (PNO-P-Rate), the decision variables include: (i) the continuous rate allocation variables $\left\{R_{0}^{k}\right\}$, and (ii) the discrete sets of $\Omega_{1}$ and $\Omega_{2}$. We emphasize that Problem (PNOP-Rate) is essentially equivalent to the original PNO's Problem 
(PNO-P), because we can determine the PU's optimal transmitpower via eq. (23) and the PNO's optimal interference-caps via eq. (24) after we obtain the optimal rate allocation decisions for Problem (PNO-P-Rate).

Problem (PNO-P-Rate) is a mixed binary nonlinear programming problem and is difficult to solve in general. Nevertheless, we notice that under the given set $\Omega_{1}$ and set $\Omega_{2}$, the objective function of Problem (PNO-P-Rate) is monotonically decreasing in $\left\{R_{0}^{k}\right\}$. Inspired by this observation, we first fix $\Omega_{1}$ and $\Omega_{2}$ and make the changes of variables of $r_{0}^{k}=\eta^{k}-R_{0}^{k}, \forall k \in \Omega_{1}$ and $r_{0}^{k}=R_{0}^{k, \max }-R_{0}^{k}, \forall k \in \Omega_{2}$. Then, Problem (PNO-PRate) becomes the following problem, under the given set $\Omega_{1}$ and set $\Omega_{2}$,

$$
\begin{aligned}
& \max _{\left\{r_{0}^{k}\right\}_{k \in \mathcal{K}}} \omega \rho \sum_{k \in \Omega_{1}} B^{k} \log _{2}\left(1+\frac{1}{2^{\left(\eta^{k}-r_{0}^{k}\right) / B^{k}}-1}\right)- \\
& \alpha \sum_{k \in \Omega_{2}} \frac{\left(2^{\left(R_{0}^{k, \max }-r_{0}^{k}\right) / B^{k}}-1\right) n_{0} B^{k}}{g_{0}^{k}}+C\left(\Omega_{1}\right) \\
& \text { subject to: } 0 \leq r_{0}^{k} \leq \eta^{k}-R_{0}^{k, \min }, \forall k \in \Omega_{1}, \\
& 0 \leq r_{0}^{k} \leq R_{0}^{k, \max }-\eta^{k}, \forall k \in \Omega_{2}, \\
& \sum_{k \in \Omega_{1}} \eta^{k}+\sum_{k \in \Omega_{2}} R_{0}^{k, \max }-\sum_{k \in \Omega_{1}+\Omega_{2}} r_{0}^{k} \geq R_{0}^{\text {req }} .
\end{aligned}
$$

Notice that Problem (PNO-P-Rate') might be infeasible, if the given sets $\Omega_{1}$ and $\Omega_{2}$ lead to $R_{0}^{\mathrm{req}}>\sum_{k \in \Omega_{1}} \eta^{k}+\sum_{k \in \Omega_{2}} R_{0}^{k, \text { max }}$. This infeasibility, fortunately, can be easily checked with. Based on the above Problem (PNO-P-Rate'), we get the following result.

Lemma 5: (Monotonic Structure of Problem (PNO-PRate')) Under the given set $\Omega_{1}$ and set $\Omega_{2}$, Problem (PNOP-Rate') is a monotonic optimization problem with respect to the decision variables $\left\{r_{0}^{k}\right\}$.

Proof: Please refer to Appendix V. Notice that to prove the monotonic structure, we make two different changes of variables within set $\Omega_{1}$ and set $\Omega_{2}$, namely, $r_{0}^{k}=\eta^{k}-R_{0}^{k}, \forall k \in$ $\Omega_{1}$, and $r_{0}^{k}=R_{0}^{k, \max }-R_{0}^{k}, \forall k \in \Omega_{2}$. The purpose is to ensure that the feasible range of new decision variables, i.e., $\left\{r_{0}^{k}\right\}$, starts from the origin, thus matching the convention of monotonic optimization problem [34].

We first provide a brief introduction to the monotonic optimization here (interested readers please refer to [34] for the details). Monotonic optimization refers to a type of mathematical programming problems that aim at maximizing a monotonic function subject to some monotonic constraints. Specifically, the monotonic structure has an important property that any level set of a monotonic function, usually called a normal set (i.e., Definition 1 provided below), can be approximated by a nested sequence of sets of polyblock (i.e., Definition 2 below). Meanwhile, the maximum of an increasing function over a polyblock is attained at a proper vertex of the polyblock (i.e., Definition 3). Exploiting the aforementioned two properties, [35] proposed a Polyblock Approximation (PA) algorithm to solve the monotonic optimization problem. Specifically, the PA algorithm includes two key steps in each round of iteration: (i) to construct a new polyblock (based on the previous one) that approximates the feasible set closer from the outside, and (ii) to find a best vertex that maximizes the objective function under the newly constructed polyblock. The iteration process continues until we find the best vertex that falls within (or close enough to) the feasible region of the optimization problem, and this best vertex can be considered as the optimal solution. Although it is still an open question to quantify the computational complexity of PA algorithm, the PA algorithm is shown to be able to solve the monotonic optimization problems efficiently [33], [34].

Definition 1: (Normal Set) A set $\mathcal{G} \subset \mathcal{R}_{+}^{n}$ is called normal if for any two points $x$ and $x^{\prime} \in \mathcal{R}_{+}^{n}$ with $x^{\prime} \leq x$, if $x \in \mathcal{G}$, then $x^{\prime} \in \mathcal{G}$ holds.

Definition 2: (Polyblock) A set $\mathcal{P} \subset \mathcal{R}_{+}^{n}$ is called a polyblock in $[a, b] \subset \mathcal{R}_{+}^{n}$ if it is the union of a finite number of boxes $[a, z], z \in \mathcal{T} \subset[a, b]$. The set $\mathcal{T}$ is called the vertex set of the polyblock. The polyblock $\mathcal{P}$ is generated by $\mathcal{T}$ exactly. Intuitively, the polyblock can be roughly considered as a union of hyper-rectangles.

Definition 3: (Proper Vertex of Polyblock) Let $\mathcal{T}$ denote the vertex set of a polyblock $P \subset R_{+}^{n}$. A vertex $v \in \mathcal{T}$ is proper if it is not dominated by any other $v^{\prime} \in \mathcal{T}$. A polyblock is fully determined by its proper vertices.

The monotonic structure of Problem (PNO-P-Rate') in Lemma 5 enables us to adopt the PA algorithm to solve Problem (PNO-P-Rate') efficiently, under the given set $\Omega_{1}$ and set $\Omega_{2}$. In the next subsection, by using the PA algorithm as a subroutine, we further propose two algorithms to solve the original Problem (PNO-P-Rate).

\section{Procedure-III: Two Proposed Algorithms to Solve Problem (PNO-P-Rate)}

1) Algorithm to Solve Problem (PNO-P-Rate): We first propose a Polyblock Approximation based Exhaustive Search (PA-ES) Algorithm to solve Problem (PNO-P-Rate) effectively, i.e., to obtain the optimal sets $\left(\Omega_{1}^{*}, \Omega_{2}^{*}\right)$ and the corresponding optimal rate allocations $\left\{R_{0}^{k *}\right\}$. The details of Algorithm (PAES) are shown on page 9 .

Specifically, Algorithm (PA-ES) enumerates all possible combinations of $\left(\Omega_{1}, \Omega_{2}\right)$ (i.e., the while-loop from line 2 to line 20). For each enumerated $\left(\Omega_{1}, \Omega_{2}\right)$ which is feasible to Problem (PNO-P-Rate'), we invoke the PA algorithm as a subroutine to solve Problem (PNO-P-Rate') in line 14 and record the best result found so far in line 17. In summary, Algorithm (PA-ES) finds the optimal solutions of Problem (PNO-P-Rate), including (i) the optimal sets $\Omega_{1}^{*}$ and $\Omega_{2}^{*}$, and (ii) the optimal rate allocations $\left\{R_{0}^{k *}\right\}$.

After obtaining the optimal sets $\left(\Omega_{1}^{*}, \Omega_{2}^{*}\right)$ and the optimal rate allocations $\left\{R_{0}^{k *}\right\}$, the PNO can determine its optimal interference-caps $\left\{Q_{0}^{k *}\right\}$ via (24) and the optimal transmitpower $\left\{p_{0}^{k *}\right\}$ for the PU via eq. (23). We thus finish solving Problem (PNO-P) completely.

2) Low-Complexity Algorithm to Solve Problem (PNO-PRate): Algorithm (PA-ES) relies on enumerating all possible combinations of $\left(\Omega_{1}, \Omega_{2}\right)$, which requires a complexity of $O\left(2^{K}\right)$ (recall that $K$ denotes the total number of APs). To further reduce this computational complexity, we propose a low-complexity algorithm, called PA with Myopic Ordering (PA-MO) Algorithm, to solve Problem (PNO-P-Rate). The 


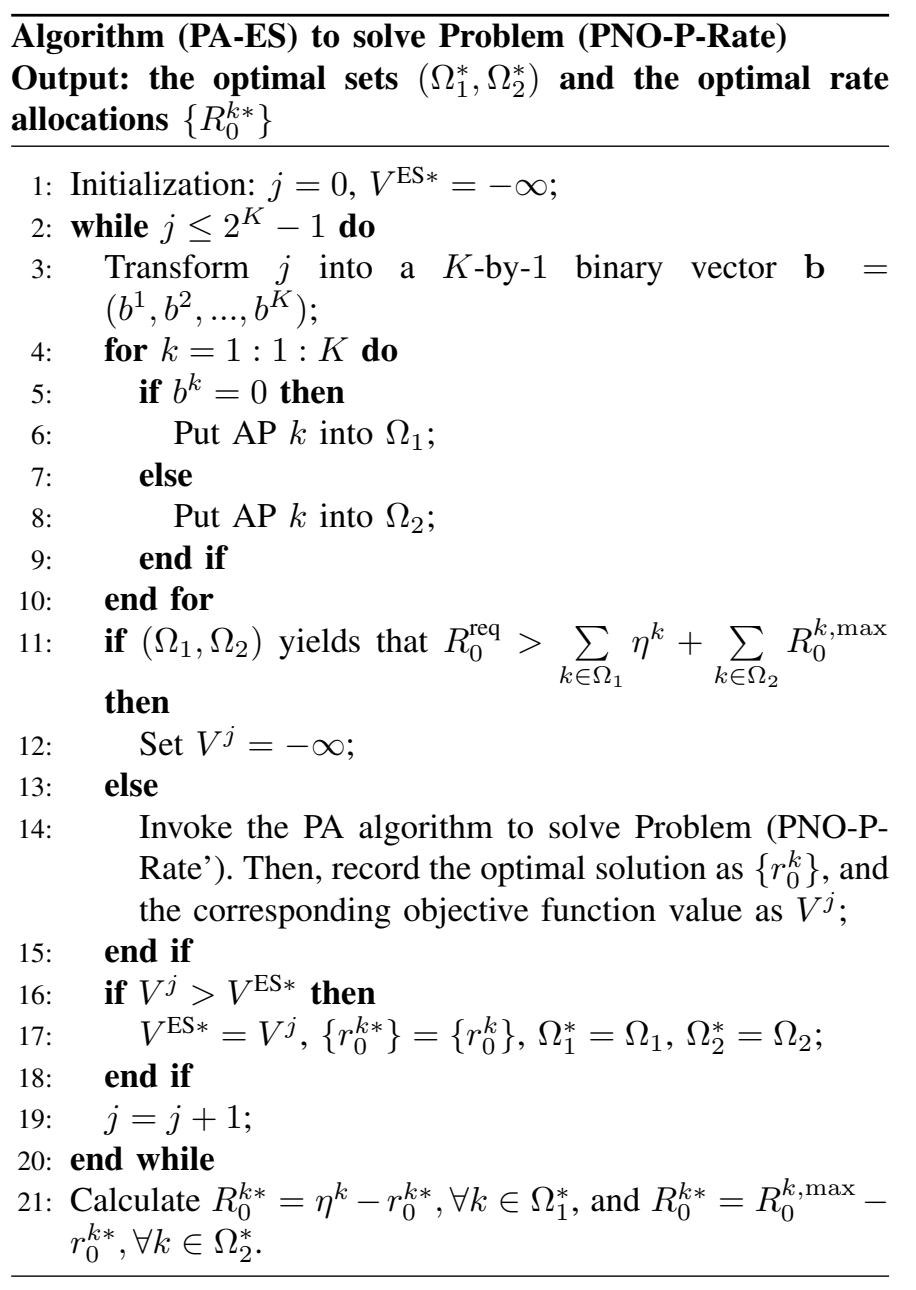

details of Algorithm (PA-MO) are also shown on page 9. Notice that we still use $\left(\Omega_{1}^{*}, \Omega_{2}^{*}\right)$ and $\left\{R_{0}^{k *}\right\}$ to denote the output of Algorithm (PA-MO) for Problem (PNO-P-Rate).

The rationale behind Algorithm (PA-MO) is as follows. Compared to using an AP with a smaller channel gain, it is more beneficial for the PNO to use an AP with a greater channel gain to accommodate the PU's throughput requirement, because it reduces the PU's need for increasing the transmitpower. Motivated by this, in Algorithm (PA-MO), we first reorder all the PNO's APs in an ascending order according to their channel gains. We then put all the APs in $\Omega_{2}$, which means that we try to use all the APs to accommodate the PU's throughput requirements at the very beginning. Then, we move the AP with the smallest channel gain from $\Omega_{2}$ to $\Omega_{1}$ (in line 4). Given the updated $\left(\Omega_{1}, \Omega_{2}\right)$ which is feasible to Problem (PNO-P-Rate'), we invoke the PA algorithm as a subroutine to solve Problem (PNO-P-Rate') (in line 8). Then, we record the best result found so far (in line 11). This process continues until we move all the APs from set $\Omega_{2}$ to set $\Omega_{1}$. Algorithm (PA-MO) requires a complexity of $O(K)$, which is less than that of Algorithm (PA-ES). As shown in the numerical results in Section VI, Algorithm (PA-MO) is still able to achieve the satisfactory results but with a further reduced computational time compared to Algorithm (PA-ES).

Summary and Implementation of the Bottom Layer Equilibrium: Till now, the PNO can use Algorithm (PA-ES) to

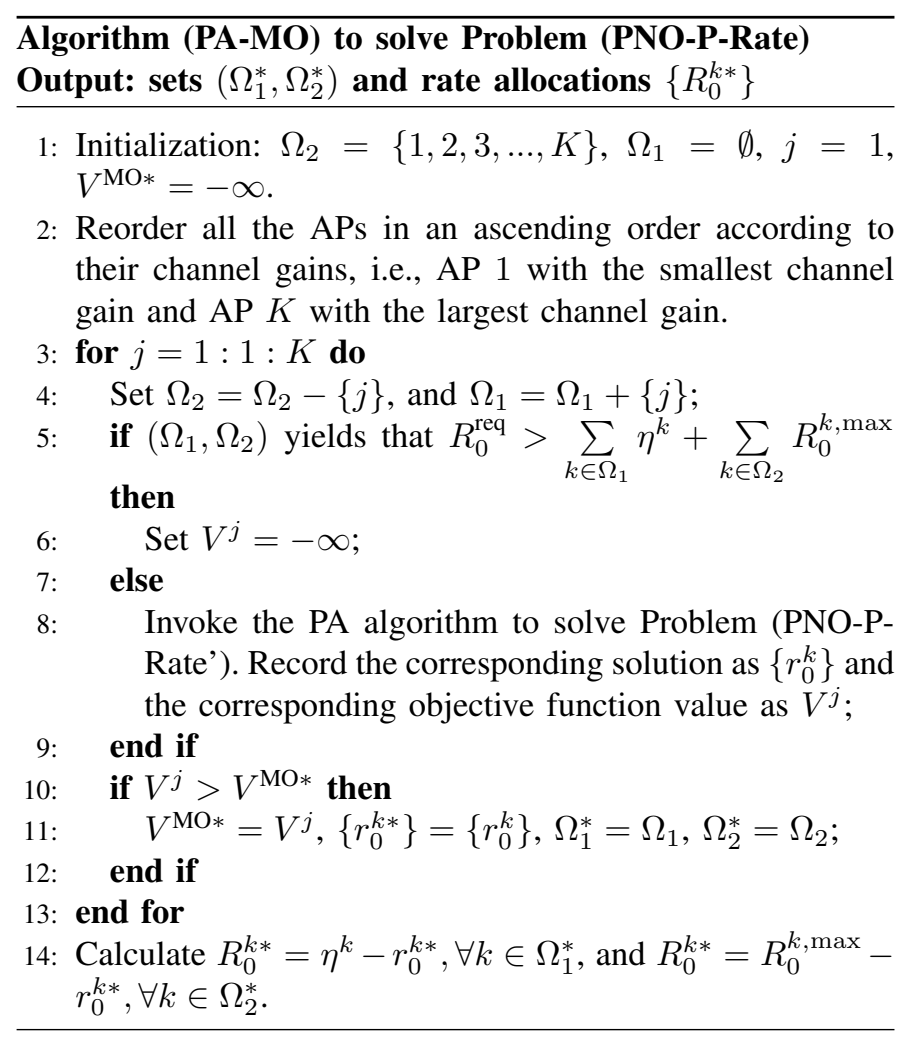

solve Problem (PNO-P-Rate) and obtain the optimal rate allocations $\left\{R_{0}^{k *}\right\}^{3}$. The PNO can further calculate its optimal interference-caps $\left\{Q_{0}^{k *}\right\}$ via eq. (24) and the PU's optimal transmit-power $\left\{p_{0}^{k *}\right\}$ via eq. (23). Knowing the PNO's optimal decisions, the SNO can subsequently compute the optimal bandwidth allocations $\left\{b_{s}^{k *}\right\}$ via eq. (14) and the transmitpower $\left\{p_{s}^{k *}\right\}$ via eq. (13) for each SU. Therefore, under a given sharing factor, the PNO and SNO eventually reach the optimal joint resource allocation decisions, which correspond to the bottom layer equilibrium.

\section{Revenue Sharing in the Top Layer Via a Nash BARGAINING GAME}

Based on the joint resource allocation decisions at the bottom layer, in this section, we continue to solve Problem (RS-P) in the top layer to determine the optimal sharing factor.

We first repeat the problem formulation below for the sake of clear presentation

$$
\text { (RS-P): } \max _{0 \leq \omega \leq 1} W(\omega)=\left(F_{0}(\omega)-F_{0}(0)\right)(S(\omega)-S(0))
$$

Recall that the PNO's revenue $F_{0}(\omega)$ is the optimal objective value of Problem (PNO-P), and the SNO's revenue $S(\omega)$ is the optimal objective value of Problem (SNO-P). Both of them are functions of the sharing factor $\omega$, and their values can be quantified based on the joint resource allocation decisions derived in the previous two sections. Meanwhile, $F_{0}(0)$ and $S(0)$ denote the respective benchmark revenues of the PNO and SNO when they fail to reach an agreement on the sharing factor (i.e., when $\omega=0$ ). Specifically, when $\omega=0$, the critical

\footnotetext{
${ }^{3}$ Alternatively, the PNO can also use Algorithm (PA-MO) to obtain the set of low-complexity solutions for its rate allocations.
} 
rate threshold $\eta^{k}$ (which is characterized in Proposition 1) is zero according to eq. (22). Correspondingly, the interferencecap $Q_{0}^{k}$ at each AP $k$ is also zero, meaning that the SUs' access is completely forbidden. Hence, we have the PNO's benchmark revenue as $F_{0}(0)=0$ and the SNO's benchmark revenue as $S(0)=0$. Problem (RP-S) means that both the PNO and SNO expect to maximize their net benefits from cooperation in a fair manner.

By substituting the joint optimal resource allocation decisions into the PNO's revenue function $F_{0}(w)$ and the SNO's revenue function $S(w)$, we can further express Problem (RS-P) in Problem (RS-P-E) as follows (the capital letter "E" stands for "Equivalence")

$$
\begin{aligned}
& \text { (RS-P-E): } \max _{0 \leq \omega \leq 1} W(\omega)= \\
& (1-\omega) \rho\left(\sum_{k} B^{k} \log _{2}\left(1+\frac{Q_{0}^{k *}}{n_{0} B^{k}+g_{0}^{k} p_{0}^{k *}}\right)\right) \times \\
& \left(\omega \rho \sum_{k} B^{k} \log _{2}\left(1+\frac{Q_{0}^{k *}}{n_{0} B^{k}+g_{0}^{k} p_{0}^{k *}}\right)-\alpha\left(\sum_{k} p_{0}^{k *}-\bar{p}_{0}\right)\right) .
\end{aligned}
$$

However, solving Problem (RS-P-E) analytically is difficult, because the optimal joint resource allocation decisions (including $\left\{p_{0}^{k *}\right\},\left\{Q_{0}^{k *}\right\},\left\{b_{s}^{k *}\right\}$ and $\left\{p_{s}^{k *}\right\}$ ) depend on the choice of sharing factor. More importantly, these joint decisions cannot be expressed in the closed-form expressions.
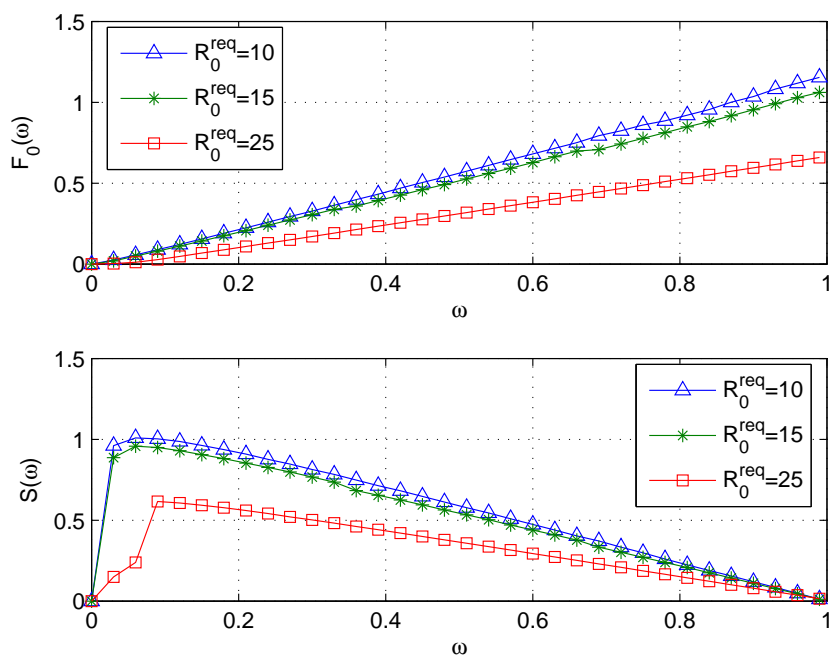

Fig. 5: Net benefits under different sharing factors. Top plot: PNO's net benefit; Bottom plot: SNO's net benefit

Before proposing an algorithmic solution for Problem (RSP), we first evaluate the effect of the sharing factor $\omega$ in Figures 5 and 6, where we assume that the PNO has four APs, and the set of PU's channel gains are $\left\{g_{0}^{k}\right\}=$ $[0.0185,0.4565,0.7919,0.8214]$ (which are randomly generated and will be described in detail in the numerical result section). We set $\alpha=0.01 \$ /$ Mbps. Figure 5 shows the PNO's net benefit (in the top plot) and the SNO's net benefit (in the bottom plot) under different sharing factors. The results show that both PNO and SNO positively benefit from the cooperation for all values of $\omega$ i.e., achieving $F_{0}(\omega)-F_{0}(0) \geq 0$ and
$S(\omega)-S(0) \geq 0$. In particular, the top plot of Figure 5 shows that the PNO's net benefit increases as the sharing factor $\omega$ increases. This result is reasonable since the PNO is the leader of the joint resource allocations in the bottom layer, and it can achieve a greater net benefit by appropriately adjusting its decisions as the sharing factor increases. In contrast, the bottom plot of Figure 5 shows that as the follower, the SNO's net benefit first increases as the sharing factor increases, and then decreases as the sharing factor is larger than a certain threshold. Intuitively, a too large sharing factor directly reduces the SNO's net benefit. While, a too small sharing factor also reduces the SNO's net benefit, since it discourages the PNO to lease its APs (i.e., by setting the interference-caps very small). Hence, to ensure that both the PNO and SNO can achieve satisfactory benefits, we need to carefully choose this sharing factor. This point is further reflected in Figure 6, which shows that the objective function $W(\omega)$ of Problem (RS-P) first increases when $\omega$ increases from zero, and then decreases when $\omega$ is above a certain threshold.

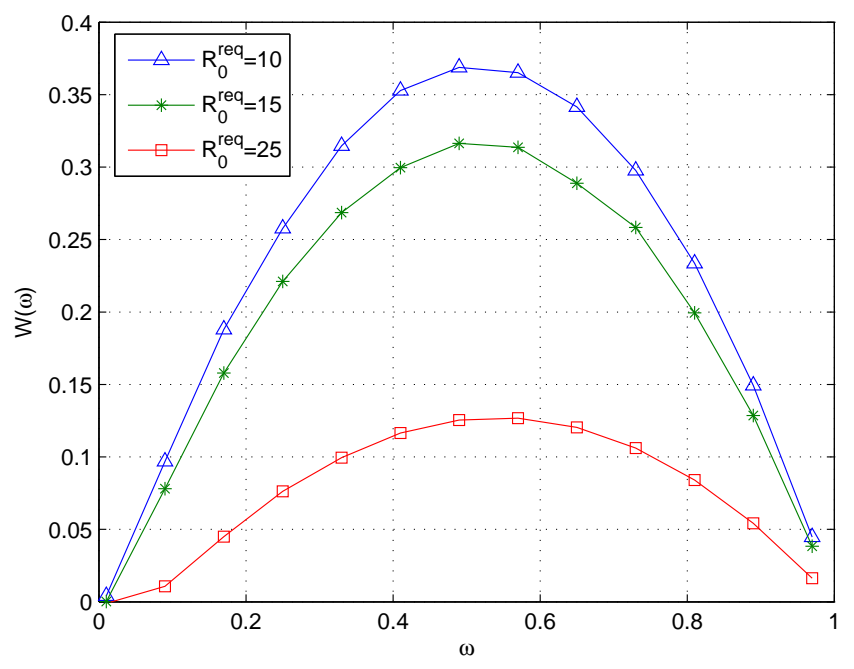

Fig. 6: Illustration of $W(\omega)$ under different throughput requirements

A keen observation from Figure 6 is that the objective function $W(\omega)$ is usually unimodal $[36]^{4}$ (notice that it is consistent with our understanding that a sharing factor that is either too small or too large will be adverse to the cooperation between PNO and SNO). This property facilitates the use of the Brent's method to find the optimal sharing factor. The Brent's method is an efficient numerical scheme to maximize a single variable function (especially the unimodal function) by combining the successive parabolic interpolation and the golden section search [36], [37]. In particular, because the Brent's method does not need any information about the firstorder derivative of the objective function, it is well suited to solving our Problem (RS-P) (and Problem (RS-P-E)), whose objective functions cannot be expressed analytically. Thus, we propose a Brent's Method based algorithm, called Algorithm

\footnotetext{
${ }^{4}$ Suppose that a function $f($.$) is defined on the interval [a, b]$, and there exists a value $c$ within $[a, b]$ strictly. Function $f($.$) is considered to be unimodal$ if it increases (or decreases) within $[a, c]$ and decreases (or increases) within $[c, b]$.
} 
(BM-RS), to solve Problem (RS-P). The details of Algorithm (BM-RS) are shown on page 11 .

We briefly introduce the Brent's method here. Interested readers please find more details in [36], [37]. In each iteration, the Brent's method tracks six critical points denoted by $a, b, x, w, v$ and $u$. Here, $a$ and $b$ denote the boundary points of the interval, within which we search for the optimal solution. $(x, w, v)$ is a triplet, where $x$ denotes the point achieving the best result so far, $w$ denotes the point achieving the second best result, and $v$ serves to record the previous value of $w$ (i.e., the value of $w$ in the previous round of iteration). Finally, $u$ denotes the next point to evaluate the objective function. The iteration process in the Brent's method includes two steps: (i) generating the new point (to be evaluated) by the parabolic interpolation (in line 11 of Algorithm (BM-RS)) or the golden section method when the parabolic interpolation is infeasible (in line 13 and line 16), and (ii) updating the boundary points $a$ and $b$ of the interval (from line 23 to line 27) and the triplet $(x, w, v)$ (in line 28) based on the newly evaluated point $u$. The detailed formulas for the parabolic interpolation and the golden section method can be found in [36], [37] and are omitted here due to space limitation. The termination conditions (in line 5) require that (i) the boundary points $a$ and $b$ are close enough, and (ii) the midpoint of interval $[a, b]$ is close enough to $x$, where $\epsilon$ denotes a small tolerance for computational error.

In particular, in lines 2, 19, and 21 of Algorithm (BM-RS), to evaluate $W(z)$ under a given $z$ (here $z$ could represent $x$, $w, v$ and $u$ ), we invoke Algorithm (PA-ES) as a subroutine to obtain the PNO's revenue $F_{0}(z)$ as well as its optimal resource allocation decisions. Then, by using the PNO's optimal resource allocation decisions, we can get the SNO's optimal decisions via eq. (13) and eq. (14). Thus, we get the value of SNO's optimal revenue $S(z)$, which, together with the value of $F_{0}(z)$, yields the value of $W(z)$. In line 11, if the parabolic interpolation is feasible, we use the parabolic interpolation to generate point $u$, which will be evaluated in line 19 and line 21 of Algorithm (BM-RS). Otherwise, we use the golden section to generate point $u$ in line 13 and line 16. After evaluating $W(u)$, we first update the boundary points $a$ and $b$ in line 24 and line 26 in order to shrink the search-interval, and then we update the triplet $(x, w, v)$ and the corresponding objective function values associated with them in line 28 .

Summary and Implementation of the Top Layer Equilibrium: Using Algorithm (BM-RS), the PNO and SNO can solve Problem (RS-P) and determine the optimal sharing factor, which corresponds to the equilibrium of the bargaining game in the top layer. More specifically, according to the computational complexity analysis in [37], Algorithm (BM-RS) is guaranteed to converge to the optimal sharing factor in an order of $O\left(\left[\log _{2}\left(\frac{\bar{\omega}-\underline{\omega}}{\varepsilon}\right)\right]^{2}\right)$ rounds of iterations, where $\varepsilon$ denotes the tolerance of computational error, and $[\underline{\omega}, \bar{\omega}]$ denotes the search interval (which is $[0,1]$ for Problem (RS-P-E)). Further substituting the optimal sharing factor back into Problem (PNO-P) and Problem (SNO-P) in the bottom layer, the PNO and SNO can determine their optimal joint resource allocation decisions

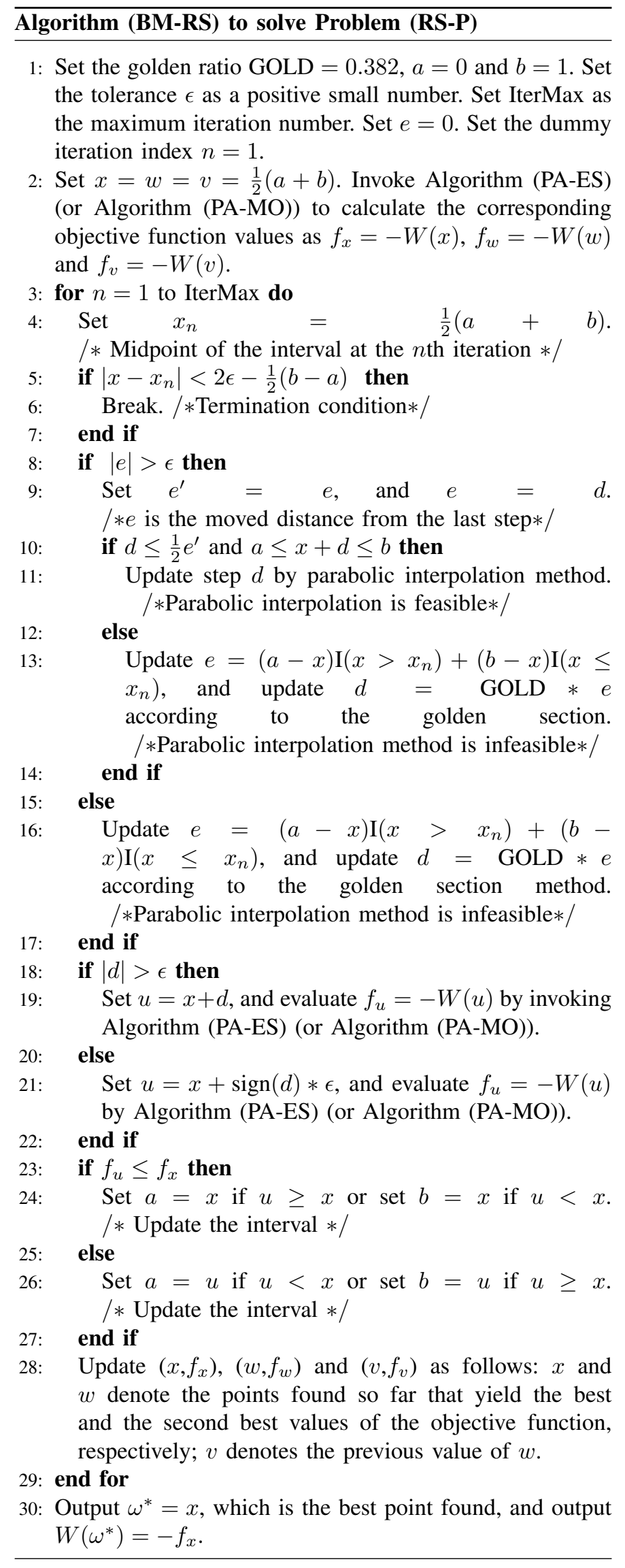


(as described in the previous Section III and Section IV) ${ }^{5}$. Thus, the PNO and SNO reach the optimal joint resource allocation decisions under their optimal revenue sharing factor, i.e., reaching the final equilibrium of the two-layered game.

\section{NUMERICAL RESULTS}

In this section, we numerically evaluate the effectiveness of our proposed algorithms and the performance of the revenue sharing based resource allocation scheme. We assume that each AP $k$ has a bandwidth $B^{k}=1 \mathrm{MHz}$. At each AP $k$, the PU's minimum throughput is $R_{0}^{k, \min }=2 \mathrm{Mbps}$, and its maximum throughput is $R_{0}^{k, \max }=15 \mathrm{Mbps}$. We use a distance-based model, which is similar to [33], to determine the channel gain from the PU (or each SU) to different APs. Specifically, the channel gain from the PU to AP $k$ is quantified by $g_{0}^{k}=\frac{\left\|\bar{g}_{0}^{k}\right\|^{2}}{\left(d_{0}^{k}\right)^{3}}$, where $d_{0}^{k}$ denotes the distance between the PU and AP $k$, and the random parameter $\bar{g}_{0}^{k}$ follows the identical and independent Gaussian distribution with zero mean and unit variance. Besides, we set the PU's marginal cost for its transmit-power consumption as $\alpha=0.01 \$ / \mathrm{W}$ and set $\left\{\beta_{s}\right\}$ as an all-one vector.

\section{A. Performance of Algorithm (PA-ES) and Algorithm (PA-MO)}
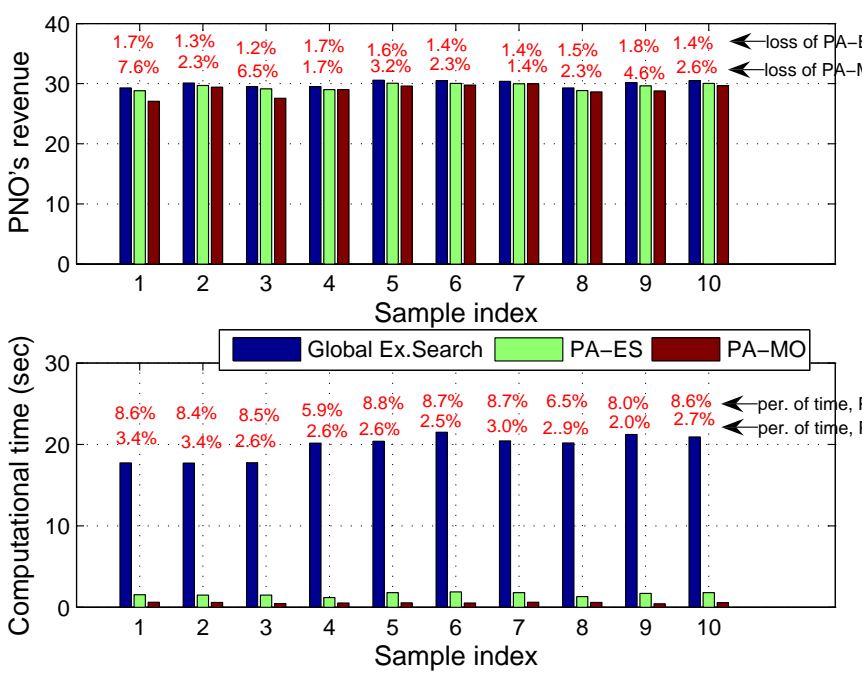

Fig. 7: Performance of Algorithm (PA-ES) and Algorithm (PA-MO) under ten sets of differently and independently generated channel gains. Top plot: PNO's revenue; Bottom plot: Computational time. We set $R_{0}^{\text {req }}=20 \mathrm{Mbps}$.

Figure 7 shows the performance of Algorithm (PA-ES) and Algorithm (PA-MO), both of which solve Problem (PNO-PRate). We fix $\omega=0.8$ and $R_{0}^{\text {req }}=20 \mathrm{Mbps}$. For the comparison purpose, we use the globally exhaustive search method to get the optimum of Problem (PNO-P-Rate). Notice that the globally exhaustive search method requires a very high computational complexity. For instance, suppose that for each AP $k$,

\footnotetext{
${ }^{5}$ Recall that in the bottom layer, for each given sharing factor $\omega$, Algorithm (PA-ES) (or Algorithm (PA-MO)) can compute $\left\{F_{0}(\omega), S(\omega)\right\}$ and the associated resource allocation decisions, which correspond to the Stackelberg game equilibrium.
}

we quantify the PNO's rate allocation into $M$ levels. Then, the globally exhaustive search requires a complexity of $\mathcal{O}\left(M^{K}\right)$. Figure 7 shows the performance of Algorithm (PA-ES), Algorithm (PA-MO), and the exhaustive search method, under ten sets of randomly and independently generated channel gains. The results show that Algorithm (PA-ES) achieves the results close to the global optimum obtained by the exhaustive method, but consuming a significantly reduced computational time. Specifically, as marked in red in Figure 7, the computational time of Algorithm (PA-ES) is less than $9 \%$ of that used by the exhaustive search method, and its relative loss compared to the exhaustive search is less than $2 \%$. Meanwhile, Figure 7 also shows that the low-complexity Algorithm (PA-MO) can further reduce the computational time compared to Algorithm (PA-ES) but still with a slight yet acceptable loss of the optimality. Specifically, the computational time of Algorithm (PA-MO) further reduces to be less than $4 \%$ of that used by the exhaustive search method, and its relative loss compared to the exhaustive search method is less than $8 \%$.
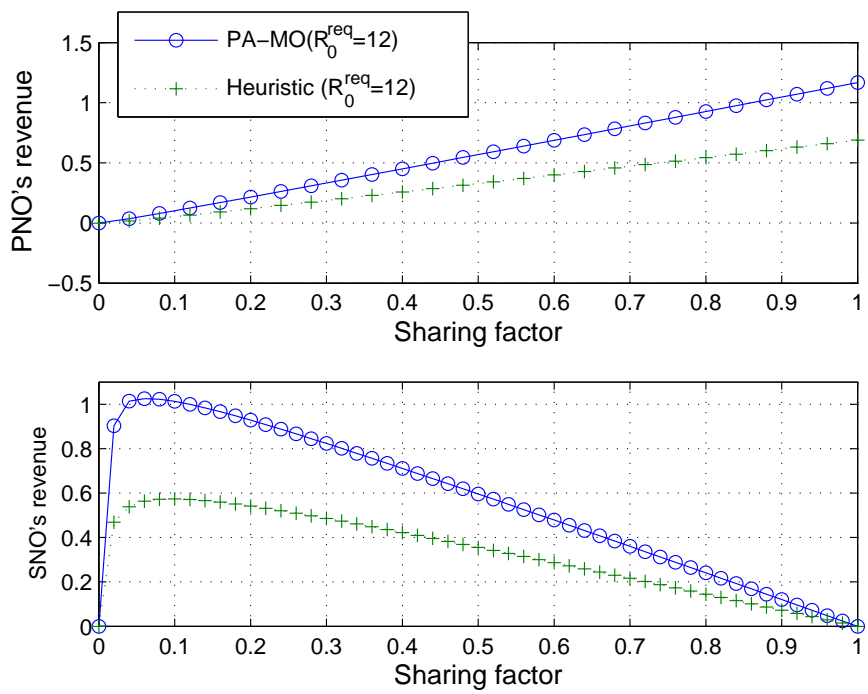

Fig. 8: Comparison between Algorithm (PA-MO) and the heuristic method under $R_{0}^{\text {req }}=12 \mathrm{Mbps}$. Top plot: PNO's revenue; Bottom plot: SNO's revenue.

In Figure 8 and Figure 9, we further compare the performance of Algorithm (PA-MO) with that of a heuristic method, which equally divides the PU's total rate requirement $R_{0}^{\text {req }}$ on all APs. Specifically, Figure 8 shows the comparison results under $R_{0}^{\text {req }}=12 \mathrm{Mbps}$, and Figure 9 shows the results under $R_{0}^{\text {req }}=15 \mathrm{Mbps}$. In Figure 8, the top plot shows that under each sharing factor, Algorithm (PA-MO) achieves a larger PNO's revenue than the heuristic method. Meanwhile, the bottom plot shows that Algorithm (PA-MO) achieves a larger SNO's revenue than the heuristic method. The similar results are also reflected in Figure 9. Moreover, a comparison between Figure 8 and Figure 9 shows that the performance gap between Algorithm (PA-MO) and the heuristic algorithm grows when the PU's throughput requirement $R_{0}^{\text {req }}$ increases. This result implies that the joint optimization of resource allocations in the bottom layer will play a more important role in improving the revenues of PNO and SNO as the PU's QoS requirement 

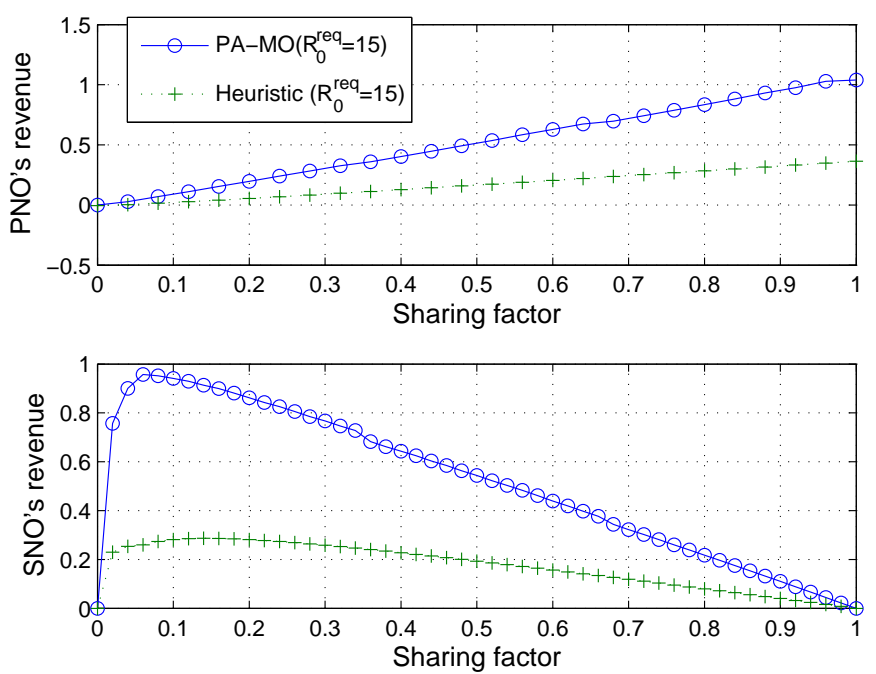

Fig. 9: Comparison between Algorithm (PA-MO) and the heuristic method under $R_{0}^{\text {req }}=15 \mathrm{Mbps}$. Top plot: PNO's revenue; Bottom plot: SNO's revenue.

becomes more stringent. In other words, the joint resource allocations without an appropriate planing could result in a larger revenue loss as the PU's QoS requirement grows.

\section{B. Performance of Algorithm (BM-RS)}
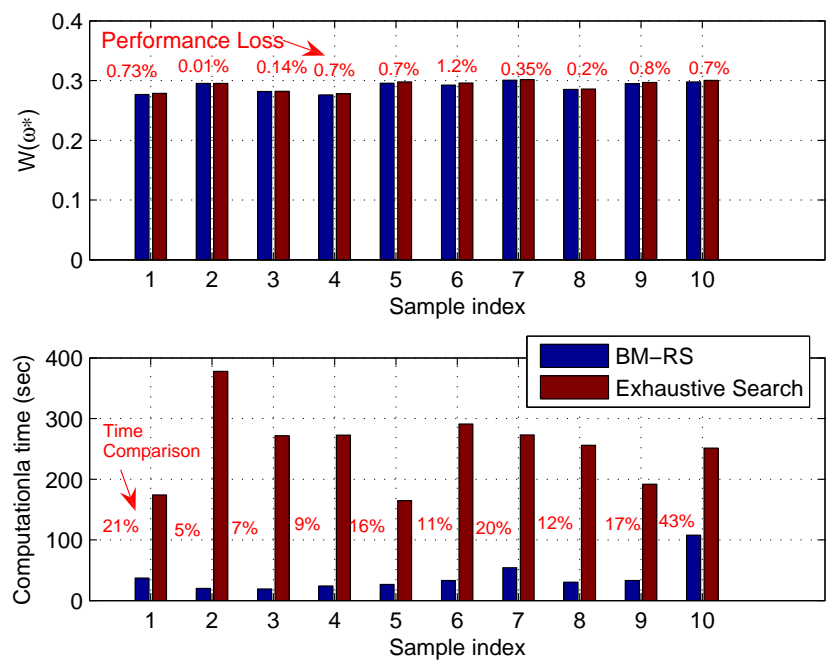

Fig. 10: Performance of Algorithm (BM-RS) under ten sets of different channel gains which are randomly and independently generated. Top plot: Value of $W\left(\omega^{*}\right)$; Bottom plot: Computational time.

We next show the performance of Algorithm (BM-RS), which solves Problem (RS-P). In Figure 10, we fix $R_{0}^{\text {req }}=$ $15 \mathrm{Mbps}$ and randomly and independently generate ten sets of different channel gains for test. As a benchmark, we use the exhaustive search method to solve Problem (RS-P) and get the globally optimal result. The top plot shows that Algorithm (BM-RS) achieves the results close to the global optimum under all the tested cases. As marked in red in the top plot, the relative loss is less than $1.5 \%$. The bottom
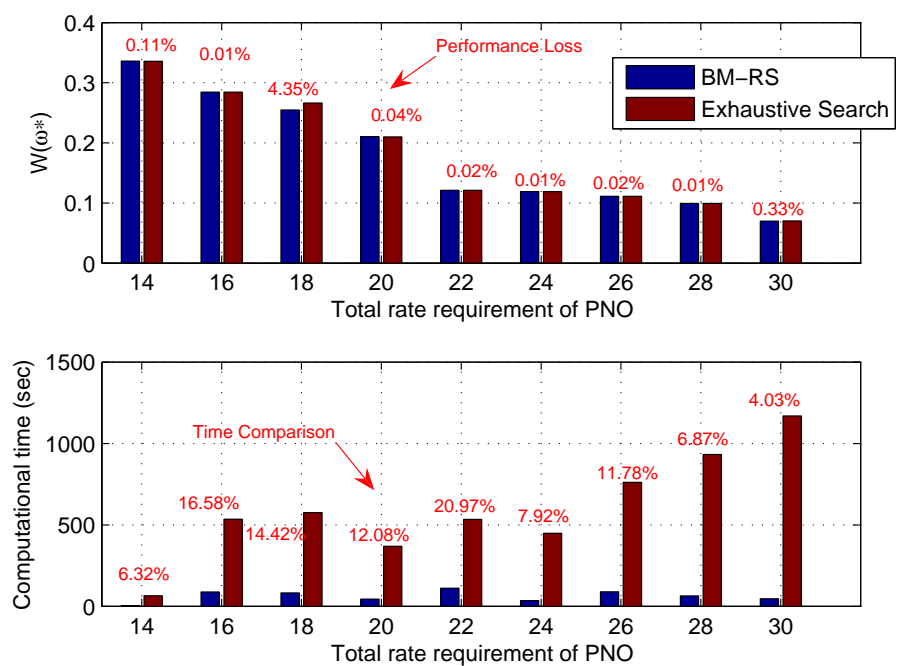

Fig. 11: Performance of Algorithm (BM-RS) under different $R_{0}^{\text {req }}$. Top plot: Value of $W\left(\omega^{*}\right)$; Bottom plot: Computational time.

plot shows that Algorithm (BM-RS) consumes a significantly less computational time than the exhaustive search method. Furthermore, Figure 11 shows the performance of Algorithm (BM-RS) under different PU's total throughput requirements, ranging from $R_{0}^{\text {req }}=14 \mathrm{Mbps}$ to $R_{0}^{\text {req }}=30 \mathrm{Mbps}$. The top plot also shows that Algorithm (BM-RS) achieves the results close to the global optimum under all the cases. As marked in red in the top plot, the relative loss is less than 4.5\%. Meanwhile, the bottom plot shows that Algorithm (BM-RS) consumes a significantly less computational time than the exhaustive search method.

\section{Net Benefits of PNO and SNO and Impact of PU's Marginal Power Cost}

Finally, in Figure 12, we show the influence of the PU's marginal power consumption cost $\alpha$ on the respective net benefits of PNO and SNO. The top plot shows that the PNO and SNO achieve positive yet almost equal net benefits, which are decreasing in $\alpha$. The almost equal net benefits of the PNO and SNO stem from the property of Nash bargaining model, which enables all agents engaged in the bargaining process to achieve the net gains in a fair manner as much as possible, depending on their respective bargaining power [32], [44] ${ }^{6}$. Meanwhile, the bottom plot shows that the optimal sharing factor increases in the PU's marginal power consumption cost. This result is consistent with our understanding that the PNO expects to get a greater portion of the SNO's revenue when it faces a greater power consumption cost. For the same reason, the optimal sharing factor also increases in the PU's total throughput requirement, which is reflected by the comparison between the two cases of $R_{0}^{\text {req }}=8 \mathrm{Mbps}$ and $R_{0}^{\mathrm{req}}=10 \mathrm{Mbps}$.

\footnotetext{
${ }^{6}$ In the top layer Nash bargaining game, we consider that the PNO and SNO have the same bargaining power, which yields the almost equal net benefits achieved by the PNO and SNO.
} 

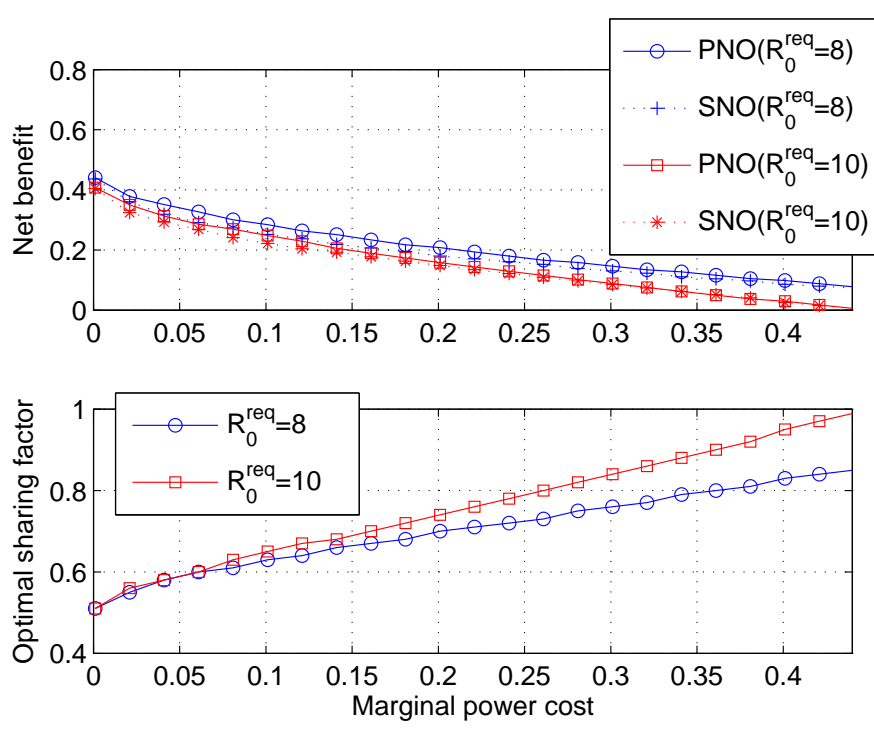

Fig. 12: Influence of the PU's marginal power consumption cost. Top plot: net benefits of the PNO and SNO; Bottom plot: the optimal sharing factor. We consider two different PNO's total rate requirements, i.e., $R_{0}^{\text {req }}=8$ and $R_{0}^{\text {req }}=10 \mathrm{Mbps}$.

\section{CONCLUSION}

In this paper, we propose a revenue sharing based resource allocation scheme for dynamic spectrum access networks. The intrinsic coupling effect between the joint resource allocations of the PNO and SNO and their revenue sharing scheme motivates us to model their interaction as a two-layered game, including the Nash bargaining game in the top layer to address their revenue sharing and the Stackelberg game in the bottom layer to address their joint resource allocation decisions. Despite the non-convexity of the joint resource allocation problem and the difficulty due to the lack of an analytical objective function for the subsequent revenue sharing optimization problem, we propose efficient algorithms to solve both the top layer and bottom layer problems, and thus obtain the optimal revenue sharing scheme and the corresponding optimal joint resource allocation decisions. Numerical results illustrate the effectiveness of our proposed algorithms and show that the revenue sharing based resource allocation scheme can enable both the PNO and SNO to achieve net benefits in a fair manner and reach a win-win situation eventually. One future direction is to incorporate the imperfect channel state information by using the robust optimization methodologies such that the proposed revenue sharing based resource allocation scheme will be robust to this imperfect information.

\section{APPENDIX I: PRoOF OF LEMMA 2}

To solve Problem (SNO-P), we relax constraint (2) and put it into the objective function by using the dual vector $\left\{\mu^{k}\right\}$. Notice that in Problem (SNO-P), the sharing factor $\omega$ and the price $\rho$ are linear and fixed scalars, which can thus be temporarily omitted in deriving the optimal solution. As a result, we can obtain the Lagrangian function $L_{1}\left(b_{s}^{k}, \mu^{k}\right)$ as

$$
\begin{array}{r}
L_{1}\left(b_{s}^{k}, \mu^{k}\right)=\sum_{k \in \mathcal{K}} \sum_{s \in S_{k}} b_{s}^{k} \log _{2}\left(1+\frac{g_{s}^{k} p_{s}^{k}}{b_{s}^{k}\left(n_{0}+g_{0}^{k} p_{0}^{k} / B^{k}\right)}\right)+ \\
\sum_{k \in \mathcal{K}} \mu^{k}\left(B^{k}-\sum_{s \in S_{k}} b_{s}^{k}\right) .
\end{array}
$$

Let $\left\{b_{s}^{k *}\right\}$ denote the optimal bandwidth allocations of Problem (SNO-P). Then, according to the Karush-Kuhn-Tucker (KKT) conditions [43], we get a sufficient condition to quantify the optimal bandwidth allocation as follows

$$
\begin{array}{r}
\log _{2}\left(1+\frac{g_{s}^{k} p_{s}^{k}}{b_{s}^{k}\left(n_{0}+g_{0}^{k} p_{0}^{k} / B^{k}\right)}\right)-\frac{1}{\ln 2} \frac{g_{s}^{k} p_{s}^{k}}{g_{s}^{k} p_{s}^{k}+b_{s}^{k^{*}}\left(n_{0}+g_{0}^{k} p_{0}^{k} / B^{k}\right)} \\
-\mu^{k}=0
\end{array}
$$

The above result can be re-expressed as

$$
f\left(\frac{g_{s}^{k} p_{s}^{k}}{b_{s}^{k^{*}}\left(n_{0}+g_{0}^{k} p_{0}^{k} / B^{k}\right)}\right)=\mu^{k}, \forall s \in \mathcal{S}_{k},
$$

with function $f(x)=\log _{2}(1+x)-\frac{1}{\ln 2}\left(\frac{x}{1+x}\right)$, which is a monotonically increasing function. These results mean that for two different SUs in $\mathcal{S}_{k}$ (let us say SU $i$ and SU s), the optimal bandwidth allocations to them should meet

$$
\frac{g_{s}^{k} p_{s}^{k}}{b_{s}^{k^{*}}\left(n_{0}+g_{0}^{k} p_{0}^{k} / B^{k}\right)}=\frac{g_{i}^{k} p_{i}^{k}}{b_{i}^{k^{*}}\left(n_{0}+g_{0}^{k} p_{0}^{k} / B^{k}\right)} .
$$

Also notice that constraint (2) should be binding at the optimum, i.e., $\sum_{s \in \mathcal{S}_{k}} b_{s}^{k *}=B^{k}$. Thus, we can express the optimal bandwidth allocation as a function of $p_{s}^{k}$ as

$$
b_{s}^{k *}=B^{k} \frac{g_{s}^{k} p_{s}^{k}}{\sum_{s \in \mathcal{S}_{k}} g_{s}^{k} p_{s}^{k}},
$$

which is proportional to its interference incurred to AP $k$. Further let $\left\{p_{s}^{k *}\right\}$ denote the optimal power allocations of SNO to the SUs, and let $I^{k *}=\sum_{s \in \mathcal{S}_{k}} g_{s}^{k} p_{s}^{k *}$ denote the total interference to AP $k$ under the optimal power allocations. Then, the optimal bandwidth allocation to SU $s$ should satisfy

$$
b_{s}^{k *}=B^{k} \frac{g_{s}^{k} p_{s}^{k *}}{I^{k *}} .
$$

We thus finish the proof.

\section{APPENDIX II: Proof OF LEMMA 3}

Based on the fairness rule, i.e. $\frac{R_{s}^{k}}{\beta_{s}^{k}}=\frac{R_{j}^{k}}{\beta_{j}^{k}}, \forall s \neq j, s, j \in \mathcal{S}_{k}$ and each SU s's throughput given by eq. (8), we obtain that

$$
\frac{g_{s}^{k} p_{s}^{k}}{\beta_{s}^{k}}=\frac{g_{j}^{k} p_{j}^{k}}{\beta_{j}^{k}}, s \text { and } j \in \mathcal{S}_{k}, \forall k .
$$

In addition, since $p_{s}^{k}$ needs to satisfy $\sum_{s \in \mathcal{S}_{k}} g_{s}^{k} p_{s}^{k}=I^{k}$ and $I^{k}=Q_{0}^{k}$ to fully exploit the interference-cap, the power allocation under the fairness rule can be expressed as

$$
g_{s}^{k} p_{s}^{k}=\frac{\beta_{s}^{k}}{\sum_{j} \beta_{j}^{k}} Q_{0}^{k}, s \in \mathcal{S}_{k}, \forall k .
$$

The above result means that at $\mathrm{AP} k$, each $\mathrm{SU} s \in \mathcal{S}_{k}$ shares the interference-cap in a proportional way. As a result, each SU s's optimal power allocation is given by

$$
p_{s}^{k *}=\frac{\beta_{s}^{k}}{\sum_{j \in \mathcal{S}_{k}} \beta_{j}^{k}} \frac{Q_{0}^{k *}}{g_{s}^{k}}, \forall s \in \mathcal{S}_{k}, \forall k .
$$


Further combining the above result with eq. (7), the optimal bandwidth allocation becomes

$$
b_{s}^{k *}=\frac{\beta_{s}^{k}}{\sum_{j \in \mathcal{S}_{k}} \beta_{j}^{k}} B^{k}, \forall s \in \mathcal{S}_{k}, \forall k .
$$

We thus finish the proof.

\section{APPENDIX III: PROOF OF LEMMA 4}

To verify that Problem (PNO-P-E') is a strictly convex problem under the given $\left\{R_{0}^{k}\right\}$, we need to verify that the objective function is a concave function, and all the constraint functions are also concave. Apparently, the constraint function associated with each AP is linear, and thus is concave. According to [43], the positively linear combination keeps the concavity. Thus, it suffices to prove that each separable component of the objective function, i.e.,

$$
F_{2}\left(p_{0}^{k}\right)=\omega \rho B^{k} \log _{2} \frac{g_{0}^{k} p_{0}^{k}}{n_{0} B^{k}+g_{0}^{k} p_{0}^{k}}-\alpha p_{0}^{k}
$$

is a concave function. The concavity of function $F_{2}($.$) can be$ proved via verifying that its second-order derivative is negative, i.e.,

$$
\frac{d^{2} F_{2}}{d p_{0}^{k^{2}}}=-\frac{\omega \rho n_{0}\left(B^{k}\right)^{2}}{\ln 2} \frac{\left(n_{0} B^{k}+2 g_{0}^{k} p_{0}^{k}\right)}{\left(n_{0} B^{k}+g_{0}^{k} p_{0}^{k}\right)^{2}\left(p_{0}^{k}\right)^{2}}<0 .
$$

Based on the linear constraints and the concave objective function, Problem (PNO-P-E') is a strictly convex optimization problem with respect to $\left\{p_{0}^{k}\right\}$. We thus finish the proof.

\section{APPENDIX IV: ProOF OF PROPOSITION 1}

We know that: (i) Problem (PNO-P-E') is a strictly convex optimization problem with respect to $\left\{p_{0}^{k}\right\}$ (according to Lemma 4), and (ii) Problem (PNO-P-E') can be decomposed into each individual AP. Thus, we introduce the Lagrange multiplier $\lambda^{k}$ to relax constraint (21) into the objective function for each AP $k$. Accordingly, we obtain a Lagrangian function $L_{2}\left(p_{0}^{k}, \lambda^{k}\right)$ associated with AP $k$ as follows

$$
\begin{array}{r}
L_{2}\left(p_{0}^{k}, \lambda^{k}\right)=\omega \\
\omega B^{k} \log _{2} \frac{g_{0}^{k} p_{0}^{k}}{n_{0} B^{k}+g_{0}^{k} p_{0}^{k}}-\alpha p_{0}^{k}+ \\
\lambda^{k}\left(g_{0}^{k} p_{0}^{k}-\left(2^{R_{0}^{k} / B^{k}}-1\right) n_{0} B^{k}\right) .
\end{array}
$$

According to the KKT conditions [43], the primal and dual optimal solutions $\left\{p_{0}^{k *}, \lambda^{k *}\right\}$ should meet

$\lambda^{k *}\left(g_{0}^{k} p_{0}^{k^{*}}-\left(2^{R_{0}^{k} / B^{k}}-1\right) n_{0} B^{k}\right)=0$, and ${\frac{\partial L_{2}}{\partial p_{0}^{k}}}_{\mid p_{0}^{k}=p_{0}^{k *}}=0$.

From the above equations, we can obtain the optimal power allocation as follows

$$
p_{0}^{k *}=\frac{n_{0} B^{k}}{2 g_{0}^{k}}\left(\sqrt{1+\frac{4 \omega \rho g_{0}^{k}}{\left(\alpha-g_{0}^{k} \lambda^{k *}\right) n_{0} \ln 2}}-1\right) .
$$

Based on the above result, we consider the following two different cases.

Case1: If $\lambda^{k *}=0$, then we have $p_{0}^{k *}=\frac{n_{0} B^{k}}{2 g_{0}^{k}}\left(\sqrt{1+\frac{4 \omega g_{0}^{k}}{\alpha n_{0} \ln 2}}-\right.$ 1 ), which means that the optimal solution occurs within the feasible region strictly. Surprisingly, in this case, the optimal power allocation becomes independent of the rate allocation.
Case2: If $\lambda^{k *} \neq 0$, then we have $p_{0}^{k *}=\frac{\left(2^{R_{0}^{k} / B^{k}}-1\right) n_{0} B^{k}}{g_{0}^{k}}$ according to the KKT conditions, which means the optimal power allocation occurs on the boundary of the feasible region.

It is apparent that at each AP $k$, the PU's transmit-power $p_{0}^{k}$ should be lower bounded by $\frac{\left(2^{R_{0}^{k} / B^{k}}-1\right) n_{0} B^{k}}{g_{0}^{k}}$ to guarantee its throughput requirement. Hence, summarizing the above two cases, we obtain the PNO's optimal power allocation to the PU as a function of its rate allocation as

$p_{0}^{k *}\left(R_{0}^{k}\right)=\max \left\{\frac{n_{0} B^{k}}{2 g_{0}^{k}}\left(\sqrt{1+\frac{4 \omega \rho g_{0}^{k}}{\alpha n_{0} \ln 2}}-1\right), \frac{\left(2^{R_{0}^{k} / B^{k}}-1\right) n_{0} B^{k}}{g_{0}^{k}}\right\}$.

Furthermore, by setting $\frac{n_{0} B^{k}}{2 g_{0}^{k}}\left(\sqrt{1+\frac{4 \omega \rho g_{0}^{k}}{\alpha n_{0} \ln 2}}-1\right)=$ $\frac{\left(2^{R_{0}^{k} / B^{k}}-1\right) n_{0} B^{k}}{g_{0}^{k}}$, we obtain the critical threshold $\eta^{k}$ for the PU's rate allocation at AP $k$ as follows

$$
\eta^{k}=B^{k}\left(\log _{2}\left(1+\sqrt{1+\frac{4 \omega \rho g_{0}^{k}}{\alpha n_{0} \ln 2}}\right)-1\right) .
$$

Using $\eta^{k}$, we get the optimal power allocation to the PU at AP $k$ as a function of its rate allocation as

$p_{s}^{k *}\left(R_{0}^{k}\right)= \begin{cases}\frac{n_{0} B^{k}}{2 g_{0}^{k}}\left(\sqrt{1+\frac{4 \omega \rho g_{0}^{k}}{\alpha n_{0} \ln 2}}-1\right), & \text { when } R_{0}^{k} \leq \eta^{k}, \\ \frac{\left(2^{R_{0}^{k} / B^{k}}-1\right) n_{0} B^{k}}{g_{0}^{k}}, & \text { when } R_{0}^{k}>\eta^{k} .\end{cases}$

Based on the above result, the PNO's optimal interference-cap at AP $k$ can be given by

$$
Q_{s}^{k *}\left(R_{0}^{k}\right)= \begin{cases}\frac{g_{0}^{k} p_{0}^{k *}}{2^{R_{0}^{k} / B^{k}}-1}-n_{0} B^{k}, & \text { when } R_{0}^{k} \leq \eta^{k} \\ 0, & \text { when } R_{0}^{k}>\eta^{k}\end{cases}
$$

according to eq. (17). We thus finish the proof.

\section{ApPEndix V: Proof OF LEMMA 5}

Let us introduce the following two functions

$$
\begin{gathered}
F_{3}\left(\left\{r_{0}^{k}\right\}\right)=\omega \rho \sum_{k \in \Omega_{1}} B^{k} \log _{2}\left(1+\frac{1}{2^{\left(\eta^{k}-r_{0}^{k}\right) / B^{k}}-1}\right), \\
F_{4}\left(\left\{r_{0}^{k}\right\}\right)=-\alpha \sum_{k \in \Omega_{2}} \frac{\left(2^{\left(R_{0}^{k, \max }-r_{0}^{k}\right) / B^{k}}-1\right) n_{0} B^{k}}{g_{0}^{k}} .
\end{gathered}
$$

We thus can show that Problem (PNO-P-Rate') meets the following two properties.

Property 1: Functions $F_{3}\left(r_{0}^{k}\right)$ and $F_{4}\left(r_{0}^{k}\right)$ are both monotonically increasing, because their first-order derivatives are

$\frac{d F_{3}}{d r_{0}^{k}}=\frac{\omega \rho}{\left(2^{\left(\eta^{k}-r_{0}^{k}\right) / B^{k}}-1\right)}>0, \frac{d F_{4}}{d r_{0}^{k}}=\frac{\alpha n_{0} \ln 2}{2^{\left(R_{0}^{k, m a x}-r_{0}^{k}\right) / B^{k}}}>0$.

As a result, the objective function of Problem (PNO-P-Rate') is increasing, because a positive linear combination of two monotonically increasing functions is still monotonically increasing. Property 2: $\left\{r_{0}^{k}\right\}$ satisfies that (i) $0 \leq r_{0}^{k} \leq \eta^{k}-R_{0}^{k, \text { min }}, \forall k \in$ $\Omega_{1}$ and (ii) $0 \leq r_{0}^{k} \leq R_{0}^{k, \max }-\eta^{k}, \forall k \in \Omega_{2}$ which are both compact and normal sets (please refer to Definition 1 provided close to the end of Section IV.B). Meanwhile, $\left\{r_{0}^{k}\right\}$ satisfies $\sum_{k \in \Omega_{1}} \eta^{k}+\sum_{k \in \Omega_{2}} R_{0}^{k, \text { max }}-\sum_{k \in \Omega_{1}+\Omega_{2}} r_{0}^{k} \geq R_{0}^{\text {req }}$, which is also a normal set. Thus, the whole feasible set for $\left\{r_{0}^{k}\right\}$ is a normal set. 
According to [34], maximizing a monotonically increasing function over a normal set falls within the scope of monotonic optimization problem. Therefore, the above Property 1 and Property 2 together show that Problem (PNO-P-Rate') is a monotonic optimization problem. This finishes the proof.

\section{ACKNOWLEDGEMENT}

The work of $\mathrm{Y}$. Wu and Q.H. Zhu was supported, in partial, by NSFC-61303235, NSFC-61379122 and ZJNSFLQ13F010006. The work of J. Huang was supported, in partial, by the General Research Funds (Project Number CUHK 412713 and CUHK 412511) established under the University Grant Committee of the Hong Kong Special Administrative Region, China. The work of D. H.K. Tsang was supported, in partial, by the General Research Fund 619911, established under the University Grant Committee of the Hong Kong Special Administrative Region, China.

\section{REFERENCES}

[1] Y.C. Liang, K.C. Chen, G.Y. Li, and P. Mahonen, "Cognitive Radio Networking and Communications: An Overview," IEEE Transactions on Vehicular Technology, vol. 60, no. 7, pp. 3386-3407, Sept. 2011

[2] B.B. Wang and K.J.R. Liu, "Advances in Cognitive Radio Networks: A Survey," IEEE Journal of Selected Topics in Signal Processing, vol. 5, no. 1, pp. 5-23, 2011

[3] Y. Zhang, R. Yu, M. Nekovee, Y. Liu, S. Xie, and S. Gjessing, "Cognitive Machine-to-Machine Communications: Visions and Potentials for the Smart Grid," IEEE Network Magazine, vol.26, no.3, pp.6-13, May/June 2012.

[4] Q. Liang, S. Han, F. Yang, G. Sun, and X. Wang, "A DistributedCentralized Scheme for Short- and Long-Term Spectrum Sharing with a Random Leader in Cognitive Radio Networks," IEEE Journal on Selected Areas in Communications, vol. 30 no. 11, pp. 2274-2284, 2012

[5] L. Gao, X. Wang, Y. Xu, and Q. Zhang, "Spectrum Trading in Cognitive Radio Networks: A Contract-Theoretic Modeling Approach." IEEE Journal on Selected Areas in Communications, vol. 29, no. 4, pp. 843-855, 2011

[6] Y. Liu, S. Xie, R. Yu, Y. Zhang, and C. Yuen, "An Efficient MAC Protocol with Selective Grouping and Cooperative Sensing in Cognitive Radio Networks," IEEE Transactions on Vehicular Technology, vol.62, no.8, pp. 3982-3941, Oct. 2013

[7] S. Xie, Y. Liu, Y. Zhang and R. Yu, "A Parallel Cooperative Spectrum Sensing in Cognitive Radio Networks," IEEE Transactions on Vehicular Technology, vol. 59, no.8, pp.4079-4092, Oct. 2010

[8] X. Chen and J. Huang, "Evolutionary Stable Spectrum Access," IEEE Transactions on Mobile Computing, vol. 12, no. 7, pp. 1281-1293, July 2013

[9] J. Zhang and Q. Zhang, "Stackelberg Game for Utility-Based Cooperative Cognitive Radio Networks," in Proc. of ACM MoBiHoc'2009.

[10] M.X. Tao and Y. Liu, "Spectrum Leasing and Cooperative Resource Allocation in Cognitive OFDMA Networks," Journal of Communications and Networks, vol. 15, no. 1, pp. 102-110, 2013

[11] Y. Yan, J. Huang, and J. Wang, "Dynamic Bargaining for Relay-Based Cooperative Spectrum Sharin," IEEE Journal on Selected Areas in Communications, vol. 1, no. 8, pp. 1480-1493, Aug. 2013

[12] Y. Xiao, G. Bi, D. Niyato and L.A. DaSilva, "A Hierarchical Game Theoretic Framework for Cognitive Radio Networks," IEEE Journal on Selected Areas in Communications, vol. 30, no. 10, pp. 2053-2069, Nov. 2012.

[13] L. Duan, L. Gao, and J. Huang, "Cooperative Spectrum Sharing: A Contract-based Approach," IEEE Transactions on Mobile Computing, vol. 13, no. 1, pp. 174-187, Jan. 2014

[14] L. Duan, J. Huang, and B. Shou, "Duopoly Competition in Dynamic Spectrum Leasing and Pricing," IEEE Transactions on Mobile Computing, vol. 11, no. 11, pp. 1706-1719, Nov. 2012

[15] D. Niyato and E. Hossain, "Market-Equilibrium, Competitive, and Cooperative Pricing for Spectrum Sharing in Cognitive Radio Networks: Analysis and Comparison," IEEE Transactions on Wireless Communications, vol. 7, no. 11, Part 1, pp. 4273-4283, Nov. 2008.
[16] D. Niyato and E. Hossain, "A Microeconomic Model for Hierarchical Bandwidth Sharing in Dynamic Spectrum Access Networks: Distributed Implementation, Stability Analysis, and Application," IEEE Transactions on Computers, vol. 59, no. 7, pp. 865-877, July 2010.

[17] S.M. Gong, X. Chen, J. Huang and P. Wang, "On-Demand Spectrum Sharing by Flexible Time-Slotted Cognitive Radio Networks," in Proc. of IEEE GLOBECOM'2012

[18] J. Huang, R. Berry, and M.L. Honig, "Distributed Interference Compensation for Wireless Networks," IEEE Journal on Selected Areas in Communications, vol. 24, no. 5, pp. 1074-1084, May 2006

[19] H. Yu, L. Gao, X. Wang and E. Hossain, "Pricing for Uplink Power Control in Cognitive Raido Networks," IEEE Transactions on Vehicular Technology, vol. 59, no. 4, pp. 1769-1778, May 2010

[20] Z.Q. Wang, L.G. Jiang, and C. He, "A Novel Price-Based Power Control Algorithm in Cognitive Radio Networks," IEEE Communications Letters, vol. 17, no. 1, pp. 43-46, 2013

[21] S.K. Jayaweera and T. Li, "Dynamic Spectrum Leasing in Cognitive Radio Networks via Primary-Secondary User Power Control Games,' IEEE Transactions on Wireless Communications, vol. 8, no. 6, pp. 33003310, June 2009

[22] Y. Wu, T.Y. Zhang, and D.H.K. Tsang, "Joint Pricing and Power Allocation for Dynamic Spectrum Access Networks with Stackelberg Game Model," IEEE Transactions on Wireless Communications, vol. 10, no. 1, pp. 12-19, Jan. 2011

[23] L. Gao, Y. Xu and X.B. Wang, "MAP: Multiauctioneer Progressive Auction for Dynamic Spectrum Access," IEEE Transactions on Mobile Computing, vol. 10, no. 8, pp. 1144-1161, August 2011

[24] L. Gao, J. Huang, Y.J. Chen and B.Y. Shou, "An Integrated Contract and Auction Design for Secondary Spectrum Trading," IEEE Journal on Selected Areas in Communications, vol. 31, no. 3, pp. 581-592, Mar. 2013.

[25] M.N. Tehrani and M. Uysal, "Auction Based Spectrum Trading for Cognitive Radio Networks," IEEE Communications Letters, vol. 17, no. 6, pp. 1168-1171, 2013

[26] L.H. Cao, W.J. Xu, J.R. Lin, K. Niu, and Z.Q. He, "An Auction Approach to Resource Allocation in OFDM-Based Cognitive Radio Networks," in Proc. of IEEE VTC'2012 Spring

[27] J. Huang, R. Berry, and M. Honig, "Auction-Based Spectrum Sharing," Mobile Networks and Applications, vol. 11, no. 3, pp. 405-418, June 2006

[28] R. Berry, M. Honig, T. Nguyen, V. Subramanian, H. Zhou and R. Vohra, "On the Nature of Revenue-Sharing Contracts to Incentivize SpectrumSharing", in Proc. of IEEE INFOCOM'2013

[29] H. Jang, H. Lee, and Y. Yi, "On the Interaction between ISP Revenue Sharing and Network Neutrality," in Proc. of ACM CoNEXT Student Workshop'2010

[30] Q.H. Zhu, Y. Wu, D.H.K. Tsang, and H. Peng, "Cooperative Spectrum Sharing in Cognitive Radio Networks with Proactive Primary System," in Proc. of IEEE 2st International Conference on Communications in China (ICCC'2013) with the International Workshop on Internet of Thing

[31] J. Huang, V. Subramanian, R. Berry, and R. Agrawal, "Downlink Scheduling and Resource Allocation for OFDMA Systems", IEEE Transactions on Wireless Communications, vol. 8, no. 1, pp. 288-296, Jan. 2009

[32] Z. Han, Z. Ji, and K.J.R. Liu, "Fair Multiuser Channel Assignment for OFDMA Networks Using Nash Bargaining Solutions and Coalitions," IEEE Transactions on Communications, vol 53, no. 8, pp. 1366-1376, Aug. 2005

[33] L. Liu, R. Zhang and K.C. Chua, "Achieving global optimality for weighted sum-rate maximization in the K-user Gaussian interference channel with multiple antennas," IEEE Transactions on Wireless Communications, vol. 11, no. 5, pp. 1933-1945, May, 2012

[34] Y.J. Zhang, L.P Qian, and J. Huang, "Monotonic Optimization in Communication and Networking Systems," Foundation and Trends in Networking, Now Publisher, October 2013

[35] H. Tuy, "Monotonic Optimization: Problems and Solution Approaches," SIAM Journal of Optimization, vol.11, no.2, pp.464-494, 2000

[36] R.P. Brent, "Algorithms for Minimization without Derivatives," Chapter 4. Prentice- Hall, Englewood Cliffs, NJ:1973.

[37] W.H. Press, S.A. Teukolsky, "Numerical Recipes: The Art of Scientific Computing," New York: Cambridge University Press, 2007

[38] S. Gong, P. Wang, and J. Huang, "Robust Performance of Spectrum Sensing in Cognitive Radio Networks," IEEE Transactions on Wireless Communications, vol. 12, no. 5, pp. 2217-2227, May 2013

[39] K. Yang, J. Huang, Y. Wu, X. Wang, and M. Chiang, "Distributed Robust Optimization (DRO) Part I: Framework and Example," Springer Journal of Optimization and Engineering, pp. 1-33, October 2012 
[40] Y. Zhu, E.D. Anese, and G.B. Giannakis, "Distributed Optimal Beamformers for Cognitive Radios Robust to Channel Uncertainties," IEEE Transactions on Signal Processing, vol. 60, no. 12, pp. 6495-6508, Dec. 2012

[41] X.W. Gong, S.A. Vorobyov, and C. Tellambura, "Joint Bandwidth and Power Allocation in Cognitive Radio Networks under Fading Channels," in Proc. of IEEE ICASSP'2011

[42] Y. Wu, and D.H.K. Tsang, "Joint Bandwidth and Power Allocations for Cognitive Radio Networks with Imperfect Spectrum Sensing," Springer Wireless Personal Communications, vol. 57, no. 1, pp 19-31, March 2011

[43] S. Boyd and L. Vandenberghe, Convex Optimization, Cambridge University Press:2004

[44] K. Binmore, A. Rubinstein, and A. Wolinsky, "the Nash Bargaining Solution in Economic Modelling," Rand Journal of Economics, vol. 17, no. 2, pp. 176-188, Summer 1986.

[45] A. Mas-Colell, M.D. Whinston, and J.R. Green, Microeconomic Theory Oxford University Press:1995.

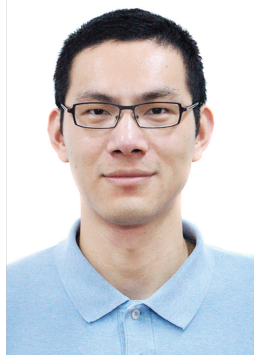

Yuan Wu (S'08-M'10) received the Ph.D degree in Electronic and Computer Engineering from the Hong Kong University of Science and Technology, Hong Kong, in 2010. He is currently an Associate Professor in the College of Information Engineering at the Zhejiang University of Technology, Hangzhou, China. He was a Postdoctoral Research Associate at the Hong Kong University of Science and Technology, Hong Kong, during 2010-2011. He was visiting scholar at Princeton University, U.S. (Aug. 2009-Jan. 2010), Georgia State University, U.S. (Jan. 2013March 2013), and Hong Kong University of Science and Technology, Hong Kong (June 2014). His research interests focus on resource allocations for cognitive radio networks and game theories with their applications in communication networks.

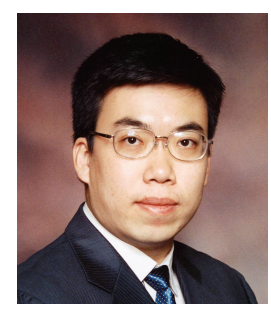

Jianwei Huang (S'01-M'06-SM'11) is an Associate Professor and Director of the Master of Science Program in the Department of Information Engineering at the Chinese University of Hong Kong. He received the Ph.D. degree in Electrical and Computer Engineering from Northwestern University in 2005, and worked as a Postdoc Research Associate at Princeton University during 2005-2007.

Dr. Huang leads the Network Communications and Economics Lab (ncel.ie.cuhk.edu.hk), with the main research focus on network optimization and games. He is the recipient of IEEE WiOPT Best Paper Award in 2014 and 2013, the IEEE SmartGridCom Best Paper Award in 2012, IEEE Marconi Prize Paper Award in Wireless Communications in 2011, the International Conference on Wireless Internet Best Paper Award 2011, the IEEE GLOBECOM Best Paper Award in 2010, Asia-Pacific Conference on Communications Best Paper Award in 2009, and the IEEE ComSoc Asia-Pacific Outstanding Young Researcher Award in 2009. He is the co-author of three recent monographs: "Wireless Network Pricing" (Morgan \& Claypool, 2013), "Monotonic Optimization in Communication and Networking Systems" (Now Publisher, 2013), and "Cognitive Mobile Virtual Network Operator Games" (Springer, 2013).

Dr. Huang serves as the Editor of IEEE Journal on Selected Areas in Communications - Cognitive Radio Series, Editor of IEEE Transactions on Wireless Communications, Guest Editor of IEEE Journal on Selected Areas in Communications special issue on "Economics of Communication Networks and Systems", Lead Guest Editor of IEEE Journal of Selected Areas in Communications special issue on "Game Theory in Communication Systems", and Lead Guest Editor of IEEE Communications Magazine Feature Topic on "Communications Network Economics". He has served as the Associate Editor-in-Chief of IEEE Communications Society Technology News (2014), the Vice Chair (2010-2012) and Chair (2012-2014) of IEEE Communications Society Multimedia Communications Technical Committee, and a Steering Committee Member of IEEE Transactions on Multimedia (2012-2014) and IEEE International Conference on Multimedia \& Expo (2012-2014). He serves as the TPC Co-Chair of NetGCoop 2014, IEEE SmartGridComm Demand Response and Dynamic Pricing Symposium 2014, IEEE GLOBECOM Selected Areas of Communications Symposium 2013, IEEE WiOpt 2012, IEEE ICCC Communication Theory and Security Symposium 2012, IEEE GlOBECOM Wireless Communications Symposium 2010, IWCMC Mobile Computing Symposium 2010, and GameNets 2009. He is a frequent TPC member of leading conferences such as INFOCOM and MobiHoc.

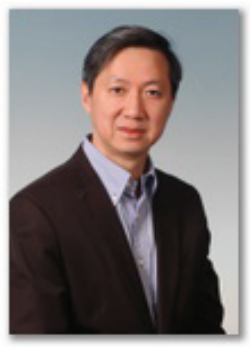

Danny H.K. Tsang (M'82-SM'00-F'12) received the $\mathrm{Ph} . \mathrm{D}$. degree in electrical engineering from the Moore School of Electrical Engineering at the University of Pennsylvania, U.S.A., in 1989. He has joined the Department of Electronic and Computer Engineering at the Hong Kong University of Science and Technology since summer of 1992 and is now a professor in the department. He was a Guest Editor for the IEEE Journal of Selected Areas in Communications' special issue on Advances in P2P Streaming Systems, an Associate Editor for the Journal of Optical Networking published by the Optical Society of America, and a Guest Editor for the IEEE Systems Journal. He currently serves as Technical Editor for the IEEE Communications Magazine. He was nominated to become an IEEE Fellow in 2012. His current research interests include Internet quality of service, $\mathrm{P} 2 \mathrm{P}$ video streaming, cloud computing, cognitive radio networks and smart grids.

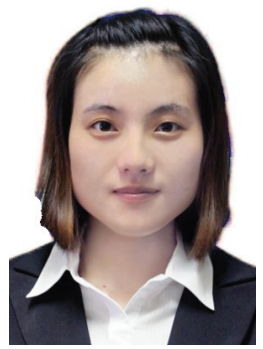

Qionghua Zhu received the B.S. degree and M.S. degree from Zhejiang University of Technology, Hangzhou, China, in 2011 and 2014, respectively. Her research interest focuses on optimal resource allocations for wireless networks, especially cognitive radio systems. 